\title{
Theoretical Study of Energy Levels and Transition Probabilities of Boron Atom
}

\author{
Zhang Tian-Yi And Zheng Neng-WU* \\ Department of Chemistry, University of Science and Technology of China, Hefei, Anhui 230026, P.R. China
}

(Received April 20, 2009)

\begin{abstract}
Though the electrons configuration for boron atom is simple and boron atom has long been of interest for many researchers, the theoretical studies for properties of BI are not systematic, there are only few results reported on energy levels of high excited states of boron, and transition measurements are generally restricted to transitions involving ground states and low excited states without considering fine structure effects, provided only multiplet results, values for transitions between high excited states are seldom performed. In this article, by using the scheme of the weakest bound electron potential model theory calculations for energy levels of five series are performed and with the same method we give the transition probabilities between excited states with considering fine structure effects. The comprehensive set of calculations attempted in this paper could be of some value to workers in the field because of the lack of published calculations for the BI systems. The perturbations coming from foreign perturbers are taken into account in studying the energy levels. Good agreement between our results and the accepted values taken from NIST has been obtained. We also reported some values of energy levels and transition probabilities not existing on the NIST data bases.
\end{abstract}

PACS numbers: 01.55.+b, 31.15.-p

\section{Introduction}

Atomic data play a very important role in many research fields such as astrophysics, laser controlled thermonuclear fusion, and physics analytical chemistry etc. Once the values of energy levels are known, many properties of atomic systems can be determined. The abundances of elements, atomic temperature, interstellar species can be inferred from the accurate knowledge of the oscillator strengths, so the development of simple and effective methods for calculating the energy levels and transition probabilities. Oscillator strengths is useful for application to various other related fields.

As a light element, BI has long been of interest and many studies of properties of BI have been carried out. Astrophysical scientists have focused their attention on the identity BI in the sun and the solar abundance of BI. As we know, accurate solar abundance determination is based on accurate values of transition rates and oscillator strengths, uncertainties in the oscillator strengths will contribute to the uncertainties in the abundance determination, so the accurate values of transition rates and oscillator strengths are useful for the astrophysical scientists' research, but because the spectrum lines of neutral boron is blended with other lines, the determination of the solar boron abundance is uncertain. Experimentally, considering the factors such as the strengths of the line, line wing and cascade effects, the experimental values of

\footnotetext{
* corresponding author; e-mail: nwzheng@ustc.edu.cn
}

boron seldom reach a precision of $20 \%$, sometimes only are estimated to be accurate about $50 \%$.

From a theoretical point of view the boron atom is an ideal system to be studied. The ground configuration is $1 s^{2} 2 s^{2} 2 p$ with three electrons outside a compact $k$-shell core, the small number of electrons makes the theoretical calculations simple, and accurate results are able to be obtained.

In recent years, attention is also concentrated on excited states that are formed by excitation of $2 p$ electron from ground states $1 s^{2} 2 s^{2} 2 p$ of boron atom, because the $1 s^{2} 2 s^{2} n l$ excited state is similar to a one-electron system with $[\mathrm{He}] 2 s^{2}$ core. However, the studies of atom boron are not systematic, there are only few results reported on energy levels of high excited states of boron, and current transition measurements are generally restricted to transitions involving ground states and low excited states without considering fine structure effects, provide only multiplet results, values for transitions between high excited states are seldom performed.

Experimentally, in 1966, with the phase-shift method, radiative lifetimes of fourteen of the uv multiplets in BI were measured by Lawrence et al., and the accuracy of their results was estimated to be $10-20 \%$ [1]. In 1969, seven mean lines were determined for excited neutral and ionic states of boron by Andersen by using the foil-excitation technique [2]. By using a hollow-cathode light source to produce the uv spectrum of BI, Goorvitch et al. measured the transitions $2 s^{2} 2 p^{2} P-2 s^{2} n d{ }^{2} D(n=3-7)$, $2 s^{2} 2 p^{2} P-2 s^{2} n s{ }^{2} S(n=3-5)$, and $2 s^{2} 2 p{ }^{2} P-2 s 2 p^{2}{ }^{2} S$ in 1972 [3]. Roig et al. studied the absorption spectrum of 
BI by flash pyrolysis technique and observed transitions from both the $2 s^{2} 2 p{ }^{2} P$ ground state and $2 s 2 p^{2}{ }^{4} P$ state in 1976 [4]. In 1979, by using the projective electron spectroscopy method, the ejected-electron spectra of highly excited autoionizing levels of BI have been studied by Rødbro et al. [5]. In 1987, for the first time the laser spectroscopic techniques have been used to investigate the neutral boron by Bergström et al. and the radiative lifetimes for the $3 p{ }^{2} P$ and $4 p{ }^{2} P$ states have been reported in their work [6]. In the course of investigating the ionization spectroscopy of boron-containing radicals, new $n p(n=5-9)$ Rydberg states were discovered by Irikura et al. in 1992 [7]. In the same year, Lynam et al. obtained the spectra of boron in a laser produced plasma experiment and predicted transition energies, oscillator strengths and intensities for the $1 s^{2} 2 s^{2} 2 p^{2} P-1 s 2 s^{2} 2 p n p$, $n \geq 2$ of BI [8]. In 2001, Glab et al. reported ${ }^{11} \mathrm{~B}$ $2 s^{2} 3 s-2 s^{2} n p(n=30-69)$ transition energies [9]. In the same year, radiative lifetimes in the $s$ and $d$ sequences of neutral boron were investigated both experimentally and theoretically by Lundberg et al., they first measured the radiative lifetimes in $\mathrm{BI} 2 s^{2} n s{ }^{2} S(n \leq 7)$ and $2 s^{2} n d{ }^{2} D$ $(n \leq 6)$ employing selective laser and then using multiconfiguration Hartree-Fock (MCHF) method to make theoretical calculations [10].

There are also many theoretical methods developed to obtain the energy levels and transition probabilities of atoms and ions. Among all the methods, MCHF method or MCHF combined with other method are the ones that are most widely used in calculating energy levels and transition probabilities of BI. In 1978, Dankwort and Trefftz used a MCHF ansatz with all Breit-Pauli (BP) corrections to calculate transition probabilities of very highly ionized boron-like series up to $\mathrm{Fe}^{21+}[11]$. After two years, the relativistic multiconfigurational DiracHartree-Fock (MCDHF) method were used to study the oscillator strengths and transition energies for E1 and M1 transitions in boron by Samii et al. [12]. In 1994, with the MCHF method, calculations have been performed for $1 s^{2} 2 s^{2} n s^{2} S n=3-6,1 s^{2} 2 s^{2} n p^{2} P n=2-6,1 s^{2} 2 s^{2} n d{ }^{2} D$ $n=3-5$ states by Carlsson et al.; in this work, they used increasing active set to calculate the transition matrix, the largest one is $7 s 6 p 5 d 4 f 3 g$ [13]. In 1996, Jönsson et al. used MCHF approach combined with configuration interaction (CI) approach to study the transition probabilities for allowed $2 s^{2} 2 p^{n}-2 s 2 p^{n+1}$ transitions in BI [14], after 4 years the same group used the BP Hamiltonian to calculate the configuration interaction in MCHF method, and applications are presented for the B-like spectrum [15], then they extend this method to study the Be-like to Ne-like sequences [16], today's computer power make the complex calculation process possible. From the work mentioned above we can see that in the MCHF method the completeness of the set of configurations used determine the accuracy of results, and the possible configurations are so many that only some can be selected for practice which limits the accuracy of the method, the more accurate the more configurations are needed and the more complex the calculation will be. There are also many other method for BI study, such as nuclear-charge-expansion (NCE) method, CI method, superposition of configurations (SOC) method, Stieltjes imaging (SI) method, multiconfiguration frozen-core (MC frozen-core) method, multichannel quantum defect theory (MQDT) and many-body perturbation theory (MBPT) [17-23], take MQDT for example the complexity of calculation rises quickly as the increase of the number of channels included in.

Since the weakest bound electron potential model (WBEPM) theory is presented, many studies have been performed to study the atomic properties for many systems [24-37]. In the present work, we investigate the energy levels, transition probabilities and oscillator strengths for BI using WBEPM theory, the results are compared with the experimental values and the results from other methods.

\section{Theory and method}

The WBEPM theory was suggested by one of the authors [38-41]. The WBEPM theory is based on the followings: (1) the considerations of successive ionization of free particles (atom and molecule); (2) the choice of zero of energy in quantum mechanics; (3) the separation of the weakest bound electron (WBE) and nonweakest bound electrons (NWBE).

In the process of successive ionization the electrons are pealed off one by one, the WBE is the most active electron in a given system, and also most easily excited or ionized, and all other electrons in the system are called NWBE. The $K$ electrons in the $K$-electrons system play sooner or later as a WBE in the ionization procedure, by removed of the first, second ... $K$-th WBE, the $K$ electrons system can give rise to $K$ stage of ionization, each stage of successive ionization processes corresponds to the remove of a WBE from the corresponding subsystem. There is only one WBE which will be removed during the ionization process, other electrons called NWBE will not be removed during the ionization process, so in terms of ionization, the WBE differs in behavior from the NWBE of the present system, so we can separate the WBE and the NWBE, and the problem of a many-electron system can be treated as one-electron problems of $K$ WBEs.

The single-electron Schrödinger equation of the WBE $i$ is (in this paper, all the energy terms in expressions are in Hartree units)

$$
\left[-\frac{1}{2} \nabla_{i}^{2}+V\left(r_{i}\right)\right] \psi_{i}=\varepsilon_{i} \psi_{i}
$$

We supposed that the WBE moves in the central potential field due to the ion-core formed nucleus and NWBEs. Considering the effect of penetrations, polarization and shielding, we suggest the potential function of WBE $i$ is

$$
V\left(r_{i}\right)=\frac{-Z^{\prime}}{r_{i}}+\frac{d(d+1)+2 d l}{2 r_{i}^{2}},
$$

where $Z^{\prime}$ is the effective nuclear charge, $l$ is the angular 
quantum number of the weakest bound electron and $d$ is a parameter which modifies the integral quantum number $n_{i}$ and angular quantum number $l_{i}$ into nonintegral $n_{i}^{\prime}$ and $l_{i}^{\prime}$.

Substituting Eq. (2) into Eq. (1), and solving the Schrödinger equation of the WBE $i$, one can obtain the expression of energy eigenvalue of WBE $i$ :

$$
\varepsilon=-\frac{Z^{\prime 2}}{2 n^{\prime 2}}
$$

where $n^{\prime}$ is the effective principal quantum number with $n^{\prime}=n+d$.

Now let us use the WBEPM theory to study atomic levels of the excited states of BI. The electronic configuration of $\mathrm{BI}$ in its ground state is $1 s^{2} 2 s^{2} 2 p$. The $2 p$ electrons are excited easily. The $n l$ electron in the excited states $1 s^{2} 2 s^{2} n l$ formed by excitation of $2 p$ electron can be assigned as WBE. Exciting WBE to various orbitals will produce various series of electronic configurations, each configuration may derive several terms and each term may go on to produce different fine structures. So the concept of spectrum-level-like series has been introduced to classify the energy levels [42]. Aspectrum-level-like series is a series that is composed of energy levels with the same spectral level symbol in a given electronic configuration series of a system. From the definition, take BI for example, the series B $1 s^{2} 2 s^{2} n d^{2} D_{3 / 2}$ is a spectrum-level-like series.

In a given spectrum-level-like series, energy levels depend on the principal quantum number of the weakest bound electron only

$$
T(n) \approx T_{\lim }-\frac{Z^{\prime 2}}{2 n^{\prime 2}}=T_{\lim }-\frac{Z^{\prime 2}}{2(n+d)^{2}} .
$$

Because the WBE moving in the field of the ion-core is somewhat analogous to the valence electron in alkali metals, and the quantum defect theory (QDT) provides a feasible way to study levels in high Rydberg states in alkali metals, we employ the representation of energy in QDT to do the transformation

$$
\frac{Z^{\prime}}{n+d}=\frac{Z_{\text {net }}}{n-\delta_{n}} \text {. }
$$

Then we get

$$
T(n)=T_{\lim }-\frac{Z_{\text {net }}^{2}}{2\left(n-\delta_{n}\right)^{2}} .
$$

For neutral atoms the net nuclear charge $Z_{\text {net }}=1$ and $\delta_{n}$ equal to the quantum defect in QDT Martin's [43] expression is used to determine $\delta_{n}$ :

$$
\delta_{n}\left(\varepsilon_{n}\right)=a_{1}+a_{2} m^{-2}+a_{3} m^{-4}+a_{4} m^{-6},
$$

in which $m=n-\delta_{0}$ and $\delta_{0}$ is the quantum defect of the lowest level in a given level series.

It should be noted that formula (5) is only for unperturbed series, but to our knowledge, for BI atom, the series $1 s^{2} 2 s^{2} n s{ }^{2} S$ are perturbed by state $1 s^{2} 2 s 2 p^{2}{ }^{2} S$ strongly, so the perturbations must be taken into account when calculating the energy levels. On the basis of Martin's and Langer's [44] works, we proposed an expression for calculating levels which are perturbed by foreign levels [25]:

$$
\delta_{n}\left(\varepsilon_{n}\right)=\sum_{i=1}^{4} a_{i} m^{-2(i-1)}+\sum_{j=1}^{P} \frac{b_{j}}{m^{-2}-\varepsilon_{j}}
$$

with

$$
\begin{aligned}
m & =n-\delta_{0}, \\
\varepsilon_{j} & =\frac{2\left(T_{\lim }-T_{j, \text { perturber }}\right)}{Z_{\text {net }}^{2}} .
\end{aligned}
$$

Here, $T_{j \text {,perturber }}$ is the energy of perturbing levels, and $P$ is the number of the foreign perturbing levels. We used the first $(4+P)$ experiment data to fit the $a_{i}$ and $b_{i}$ by least-squares method, then the higher ones in a series can be predicted with these parameters.

The transition probability of $\left(n_{f}, l_{f}\right)$ to $\left(n_{i}, l_{i}\right)$ for spontaneous emission $\left(E_{f}>E_{i}\right)$ is

$$
\begin{aligned}
& A_{f i}=\frac{4}{3} \alpha^{3}\left(E_{f}-E_{i}\right)^{3}\left|\left\langle n_{f} l_{f}|r| n_{i} l_{i}\right\rangle\right|^{2}\left(2 L_{f}+1\right) \\
& \times\left(2 L_{i}+1\right)\left(2 J_{i}+1\right) l_{>} \times W^{2}\left(l_{i} L_{i} l_{f} L_{f} ; L_{\mathrm{c}} 1\right) \\
& \quad \times W^{2}\left(L_{i} J_{i} L_{f} J_{f} ; S 1\right)
\end{aligned}
$$

in a.u. [45] where $l_{>}=\max \left(l_{f}, l_{i}\right), \alpha$ is the fine structure constant, $E_{f}$ and $E_{i}$ (in Hartree unit) are the energies of $\left(n_{f}, l_{f}\right)$ and $\left(n_{i}, l_{i}\right)$, respectively, $L_{\mathrm{c}}$ is the total orbital angular momentum of atomic core and $W(a b c d ; e f)$ is the Racah coefficient [46].

The matrix elements $\left\langle n_{f} l_{f}\left|r^{k}\right| n_{i} l_{i}\right\rangle$ can be derived as [47]:

$$
\begin{aligned}
& \left\langle n_{f} l_{f}\left|r^{k}\right| n_{i} l_{i}\right\rangle=(-1)^{n_{f}+n_{i}+l_{f}+l_{i}}\left(\frac{2 Z_{f}^{\prime}}{n_{f}^{\prime}}\right)^{l_{f}^{\prime}}\left(\frac{2 Z_{i}^{\prime}}{n_{i}^{\prime}}\right)^{l_{i}^{\prime}} \\
& \times\left(\frac{Z_{f}^{\prime}}{n_{f}^{\prime}}+\frac{Z_{i}^{\prime}}{n_{i}^{\prime}}\right)^{-l_{f}^{\prime}-l_{i}^{\prime}-k-3}\left[\frac{n_{f}^{\prime 4} \Gamma\left(n_{f}^{\prime}+l_{f}^{\prime}+1\right)}{4 Z_{f}^{\prime 3}\left(n_{f}-l_{f}-1\right) !}\right]^{-1 / 2} \\
& \times\left[\frac{n_{i}^{\prime 4} \Gamma\left(n_{i}^{\prime}+l_{i}^{\prime}+1\right)}{4 Z_{i}^{\prime 3}\left(n_{i}-l_{i}-1\right) !}\right]^{-1 / 2} \sum_{m_{1}=0}^{n_{f}-l_{f}-1} \sum_{m_{2}=0}^{n_{i}-l_{i}-1} \\
& {\left[\frac{(-1)^{m_{2}}}{m_{1} ! m_{2} !}\left(\frac{Z_{f}^{\prime}}{n_{f}^{\prime}}-\frac{Z_{i}^{\prime}}{n_{i}^{\prime}}\right)^{m_{1}+m_{2}}\left(\frac{Z_{f}^{\prime}}{n_{f}^{\prime}}-\frac{Z_{i}^{\prime}}{n_{i}^{\prime}}\right)^{-m_{1}-m_{2}}\right.} \\
& \times \Gamma\left(l_{f}^{\prime}+l_{i}^{\prime}+m_{1}+m_{2}+k+3\right) \\
& \times \sum_{m_{3}=0}^{s}\left(\begin{array}{c}
l_{i}^{\prime}-l_{f}^{\prime}+k+m_{2}+1 \\
n_{f}^{\prime}-l_{f}^{\prime}-1-m_{1}-m_{3}
\end{array}\right) \\
& \times\left(\begin{array}{c}
l_{f}^{\prime}-l_{i}^{\prime}+k+m_{1}+1 \\
n_{i}^{\prime}-l_{i}^{\prime}-1-m_{2}-m_{3}
\end{array}\right) \\
& \times\left(\begin{array}{c}
l_{i}^{\prime}+l_{f}^{\prime}+k+m_{1}+m_{2}+m_{3}+2 \\
m_{3}
\end{array}\right),
\end{aligned}
$$

where $S=\min \left(n_{f}-l_{f}-1-m_{1}, n_{i}-l_{i}-1-m_{2}\right)$ and $k>-l_{f}^{\prime}-l_{i}^{\prime}-3$.

If we let $k=1$ and $i=f$, the following equation can be derived from Eq. (10), we can get the radial expectation 
value of the $\mathrm{WBE}$

$$
\langle r\rangle_{n l}=\frac{3 n^{\prime 2}-l^{\prime}\left(l^{\prime}+1\right)}{2 Z^{\prime}} .
$$

Equations (4a) and (11) constitute a set of coupled equations

$$
\left\{\begin{array}{l}
T(n) \approx T_{\lim }-\frac{Z^{\prime 2}}{2 n^{\prime 2}}, \\
\langle r\rangle=\frac{3 n^{\prime 2}-l^{\prime}\left(l^{\prime}+1\right)}{2 Z^{\prime}} .
\end{array}\right.
$$

$T_{\lim }$ and $T(n)$ can be taken from the experimental data; in this paper they are taken from NIST website [48]. The $\langle r\rangle$ value can be calculated from many theoretical methods such as Roothanna Hartree-Fock (RHF), Hartree-Kohn-Sham (HKS), multiconfiguration Hartree-Fock (MCHF), self-interaction-corrected local spindensity (SIC-LSD), time-dependent Hartree-Fock (TDHF), Hartree-Slater and numerical Coulomb approximation (NCA) etc. [49-55]. In this paper NCA is employed to evaluate $\langle r\rangle$. NCA is a good approximation for excited states, its producing is simple and its results agree well with other theoretical methods.

After obtaining the values of $Z^{\prime}, n^{\prime}$ and $l^{\prime}$, the matrix element in Eq. (9) can be calculated, transition probabilities between two levels $\left(n_{f}, l_{f}\right)$ and $\left(n_{i}, l_{i}\right)$ can be calculated further.

\section{Results and discussion}

Energy levels, transition probabilities and oscillator strengths of atomic BI are studied, some of the results are listed here.

The energy levels for five spectrum-level-like series $1 s^{2} 2 s^{2} n s{ }^{2} S_{1 / 2}, 1 s^{2} 2 s^{2} n d{ }^{2} D_{3 / 2}, 1 s^{2} 2 s^{2} n d{ }^{2} D_{5 / 2}$, $1 s^{2} 2 s^{2} n f \quad{ }^{2} F_{5 / 2}$, and $1 s^{2} 2 s^{2} n f{ }^{2} F_{7 / 2}$ are listed in Tables $\mathrm{I}-\mathrm{V}$, and for space reason when $\mathrm{n}>30$ some results are omitted. Experimental data are listed in Tables I-V for comparison. In Table I and Table II, we list the results calculated by using multi-channel quantum defect theory (MQDT) by Liang nad Wang [22]; in their work, the energy levels for $1 s^{2} 2 s^{2} n s{ }^{2} S_{1 / 2}(n=$ $2-25)$ and $1 s^{2} 2 s^{2} n d{ }^{2} D_{3 / 2}(n=2-25)$ series are given; from their work we can see that in MQDT the complexion of calculation rises quickly as the increase of the number of channels included in, relatively our calculation procedures are quite simple.

If a foreign level has the same parity and the same quantum number $J$ with a spectrum-level-like series, this foreign level will perturb some energy levels in this series, the perturbing strength is dependent on the energy difference between the perturbing level and the perturbed level. In the treatment of the series $1 s^{2} 2 s^{2} n s^{2} S_{1 / 2}$, we take into account the effects of perturbation come from the $1 s^{2} 2 s 2 p^{2}{ }^{2} S_{1 / 2}$ level. In Table I we give the results with the consideration of foreign levels of $1 s^{2} 2 s^{2} n s^{2} S_{1 / 2}$ series, in order to take a comparison we also give the results without the perturbations. Form Table I we can see that the results without the perturbations are bad, the maximal deviation is $-358.4719(n=7)$; when we introduce the perturbations to our calculation, the results
TABLE I

Calculated results of energy levels $\left(\mathrm{cm}^{-1}\right)$ for BI $1 s^{2} 2 s^{2} n s{ }^{2} S_{1 / 2}$ (limit is $66928.10 \mathrm{~cm}^{-1}$ ) series compared with experimental data $\left(\mathrm{cm}^{-1}\right)$ and other re-

\begin{tabular}{|c|c|c|c|c|}
\hline \multirow[b]{2}{*}{$n$} & \multirow[b]{2}{*}{$T_{\exp }[48]$} & \multirow{2}{*}{$\begin{array}{c}\text { Without perturbation } \\
T_{\text {cal }}^{a}\end{array}$} & \multicolumn{2}{|c|}{ With perturbations } \\
\hline & & & $T_{\text {cal }}^{b}$ & $T_{\text {other }}[22]$ \\
\hline 3 & 40039.65 & 40039.65 & 40039.65 & 40040.76 \\
\hline 4 & 55010.181 & 55010.18 & 55010.18 & 55011.50 \\
\hline 5 & 60146.45 & 60146.45 & 60146.45 & 60145.64 \\
\hline 6 & 62482.23 & 62482.23 & 62482.23 & 62482.47 \\
\hline 7 & 64156.00 & 63797.53 & 64156.00 & 64155.59 \\
\hline 8 & 64792.07 & 64613.03 & 64767.58 & 64791.79 \\
\hline 9 & 65270.16 & 65151.20 & 65258.17 & 65270.12 \\
\hline 10 & 65609.35 & 65523.57 & 65602.67 & 65609.48 \\
\hline 11 & & 65791.18 & 65851.41 & 65855.68 \\
\hline 12 & & 65989.59 & 66036.42 & 66039.28 \\
\hline 13 & & 66140.58 & 66177.63 & 66179.63 \\
\hline 14 & & 66258.05 & 66287.82 & 66289.27 \\
\hline 15 & & 66351.19 & 66375.43 & 66376.50 \\
\hline 16 & & 66426.24 & 66446.22 & 66447.03 \\
\hline 17 & & 66487.60 & 66504.24 & 66504.86 \\
\hline 18 & & 66538.38 & 66552.38 & 66552.86 \\
\hline 19 & & 66580.88 & 66592.76 & 66593.14 \\
\hline 20 & & 66616.80 & 66626.97 & 66627.27 \\
\hline 21 & & 66647.43 & 66656.20 & 66656.43 \\
\hline 22 & & 66673.76 & 66681.37 & 66681.55 \\
\hline 23 & & 66696.56 & 66703.20 & 66703.34 \\
\hline 24 & & 66716.42 & 66722.26 & \\
\hline 25 & & 66733.84 & 66738.99 & 66739.07 \\
\hline 26 & & 66749.20 & 66753.77 & \\
\hline 27 & & 66762.80 & 66766.88 & \\
\hline 28 & & 66774.91 & 66778.56 & \\
\hline 29 & & 66785.74 & 66789.02 & \\
\hline 30 & & 66795.46 & 66798.41 & \\
\hline 35 & & 66831.82 & 66833.67 & \\
\hline 40 & & 66855.05 & 66856.28 & \\
\hline 45 & & 66870.79 & 66871.65 & \\
\hline 50 & & 66881.94 & 66882.56 & \\
\hline 55 & & 66890.13 & 66890.59 & \\
\hline 60 & & 66896.31 & 66896.67 & \\
\hline 65 & & 66901.10 & 66901.38 & \\
\hline 70 & & 66904.89 & 66905.11 & \\
\hline 75 & & 66907.93 & 66908.11 & \\
\hline 80 & & 66910.41 & 66910.56 & \\
\hline 85 & & 66912.46 & 66912.58 & \\
\hline
\end{tabular}
sults $\left(\mathrm{cm}^{-1}\right)$.

$T_{\text {cal }}^{a}$ are energy levels calculated not including perturbing level. $T_{\text {cal }}^{b}$ are energy levels calculated including perturbing level. The perturbing level is $1 s^{2} 2 s 2 p^{2}{ }^{2} S_{1 / 2}\left(63560.64 \mathrm{~cm}^{-1}\right)$ which is selected from experimental values in [48].

are greatly improved, the maximal deviation is -24.4923 $(n=8)$. From a comparison of these results, we suggest that foreign levels should be taken into account. The Martin expression is not available for perturbed levels, so we derived the expression (6) to calculate the perturbed series.

The parameters $a$ and $b$ for each series are listed in Table VI, so one can easily obtain any energy levels in the series. These parameters are obtained by fitting the 
TABLE II

Calculated results of energy levels $\left(\mathrm{cm}^{-1}\right)$ for BI $1 s^{2} 2 s^{2} n d{ }^{2} D_{3 / 2}$ (limit is $66928.10 \mathrm{~cm}^{-1}$ ) series compared with experimental data $\left(\mathrm{cm}^{-1}\right)$ and other results $\left(\mathrm{cm}^{-1}\right)$.

\begin{tabular}{|c|c|c|c|}
\hline$n$ & $T_{\exp }[48]$ & $T_{\text {cal }}$ & $T_{\text {other }}[22]$ \\
\hline 3 & 54767.63 & 54767.63 & 54767.67 \\
\hline 4 & 59993.41 & 59993.41 & 59993.39 \\
\hline 5 & 62485.42 & 62485.42 & 62485.45 \\
\hline 6 & 63845.29 & 63845.29 & 63845.16 \\
\hline 7 & 64665.30 & 64665.48 & 64665.33 \\
\hline 8 & 65197.31 & 65197.44 & 65197.30 \\
\hline 9 & 65561.64 & 65561.81 & 65561.69 \\
\hline 10 & 65822.12 & 65822.21 & 65822.09 \\
\hline 11 & 66014.58 & 66014.70 & 66014.59 \\
\hline 12 & 66160.92 & 66161.01 & 66160.90 \\
\hline 13 & 66274.65 & 66274.79 & 66274.68 \\
\hline 14 & 66364.90 & 66365.01 & 66364.91 \\
\hline 15 & 66437.62 & 66437.76 & 66437.66 \\
\hline 16 & 66497.15 & 66497.28 & 66497.17 \\
\hline 17 & 66546.47 & 66546.58 & 66546.47 \\
\hline 18 & 66587.77 & 66587.88 & 66587.77 \\
\hline 19 & 66622.72 & 66622.82 & 66622.71 \\
\hline 20 & 66652.52 & 66652.64 & 66652.54 \\
\hline 21 & 66678.17 & 66678.30 & 66678.19 \\
\hline 22 & 66700.43 & 66700.53 & 66700.43 \\
\hline 23 & 66719.79 & 66719.92 & \\
\hline 24 & 66736.82 & 66736.94 & 66736.83 \\
\hline 25 & 66752.02 & 66751.95 & 66751.84 \\
\hline 26 & 66765.22 & 66765.26 & \\
\hline 27 & 66777.12 & 66777.11 & \\
\hline 28 & 66787.66 & 66787.72 & \\
\hline 29 & 66797.25 & 66797.25 & \\
\hline 30 & 66805.63 & 66805.84 & \\
\hline 31 & 66813.66 & 66813.61 & \\
\hline 32 & 66821.00 & 66820.66 & \\
\hline 33 & 66827.01 & 66827.08 & \\
\hline 34 & 66832.68 & 66832.94 & \\
\hline 35 & 66838.33 & 66838.31 & \\
\hline 36 & 66843.28 & 66843.23 & \\
\hline 37 & 66847.81 & 66847.76 & \\
\hline 38 & 66851.99 & 66851.94 & \\
\hline 39 & 66855.78 & 66855.80 & \\
\hline 40 & & 66859.37 & \\
\hline 45 & & 66873.81 & \\
\hline 50 & & 66884.13 & \\
\hline 55 & & 66891.77 & \\
\hline 60 & & 66897.58 & \\
\hline 65 & & 66902.09 & \\
\hline 70 & & 66905.68 & \\
\hline 75 & & 66908.57 & \\
\hline 80 & & 66910.94 & \\
\hline 85 & & 66912.90 & \\
\hline 90 & & 66914.54 & \\
\hline 95 & & 66915.93 & \\
\hline 100 & & 66917.12 & \\
\hline
\end{tabular}

TABLE III

Calculated results of energy levels $\left(\mathrm{cm}^{-1}\right)$ for BI $1 s^{2} 2 s^{2} n d{ }^{2} D_{5 / 2}$ (limit is $66928.10 \mathrm{~cm}^{-1}$ ) series compared with experimental data $\left(\mathrm{cm}^{-1}\right)$.

\begin{tabular}{c|c|c||c|c|c}
\hline \hline$n$ & $T_{\text {exp }[48]}$ & $T_{\text {cal }}$ & $n$ & $T_{\text {exp }}[48]$ & $T_{\text {cal }}$ \\
\hline 3 & 54767.80 & 54767.80 & 28 & 66787.66 & 66787.71 \\
4 & 59993.51 & 59993.51 & 29 & 66797.25 & 66797.24 \\
5 & 62485.58 & 62485.58 & 30 & 66805.63 & 66805.83 \\
6 & 63845.29 & 63845.29 & 31 & 66813.66 & 66813.60 \\
7 & 64665.34 & 64665.40 & 32 & 66821.00 & 66820.66 \\
8 & 65197.29 & 65197.34 & 33 & 66827.01 & 66827.08 \\
9 & 65561.64 & 65561.71 & 34 & 66832.68 & 66832.94 \\
10 & 65822.12 & 65822.12 & 35 & 66838.33 & 66838.30 \\
11 & 66014.58 & 66014.63 & 36 & 66843.28 & 66843.23 \\
12 & 66160.92 & 66160.94 & 37 & 66847.81 & 66847.76 \\
13 & 66274.65 & 66274.73 & 38 & 66851.99 & 66851.94 \\
14 & 66364.90 & 66364.97 & 39 & 66855.78 & 66855.80 \\
15 & 66437.62 & 66437.72 & 40 & & 66859.37 \\
16 & 66497.15 & 66497.24 & 45 & & 66873.81 \\
17 & 66546.47 & 66546.55 & 50 & & 66884.13 \\
18 & 66587.77 & 66587.85 & 55 & & 66891.77 \\
19 & 66622.72 & 66622.80 & 60 & & 66897.57 \\
20 & 66652.52 & 66652.62 & 65 & & 66902.09 \\
21 & 66678.17 & 66678.28 & 70 & & 66905.68 \\
22 & 66700.43 & 66700.52 & 75 & & 66908.57 \\
23 & 66719.79 & 66719.91 & 80 & & 66910.94 \\
24 & 66736.82 & 66736.92 & 85 & & 66912.90 \\
25 & 66752.02 & 66751.94 & 90 & & 66914.54 \\
26 & 66765.22 & 66765.25 & 95 & & 66915.93 \\
27 & 66777.12 & 66777.10 & 100 & & 66917.12 \\
& & & & & \\
\hline
\end{tabular}

first $(4+P)$ experiment data, by least-squares method in expressions (5) and (6), $P$ is the number of levels perturbing, so the choice of the values used to determine the parameters is very important. In this work we chose the experimental values from NIST database [48]. NIST scientists have tried their best to collect accepted data from the original sources, the values collected by NIST database are the best available data at present.

A coupled Eq. (12) is derived for transition calculations. If the energy levels $T(n)$ and the expectation values $\langle r\rangle$ are known, the parameters $Z^{\prime}, n^{\prime}$ and $l^{\prime}$ needed for transition calculations can be obtained. The values of $T(n)$ are also taken from NIST database [48] and $\langle r\rangle$ evaluated using NCA method.

The spin-allowed transition probabilities and oscillator strength lines in BI are calculated, and the results are listed in Table VII, we compared our results to NIST data [48], the accuracy rating of NIST values are given in the sixth column in Table VII. Presently the study for transitions between individual lines for BI are quite limited both experimentally and theoretically, and the existing values are generally for transitions involving ground states and lower excited states, transitions involving highly excited states are not studied by other methods. 
TABLE IV

Calculated results of energy levels $\left(\mathrm{cm}^{-1}\right)$ for BI $1 s^{2} 2 s^{2} n f{ }^{2} F_{5 / 2}$ (limit is $\left.66928.10 \mathrm{~cm}^{-1}\right)$ series compared with experimental data $\left(\mathrm{cm}^{-1}\right)$.

\begin{tabular}{c|c|c||c|c|c}
\hline \hline$n$ & $T_{\text {exp }}[48]$ & $T_{\text {cal }}$ & $n$ & $T_{\text {exp }}[48]$ & $T_{\text {cal }}$ \\
\hline 4 & 60031.03 & 60031.03 & 25 & & 66752.30 \\
5 & 62516.52 & 62516.52 & 26 & & 66765.57 \\
6 & 63866.33 & 63866.33 & 27 & & 66777.40 \\
7 & 64679.74 & 64679.74 & 28 & & 66787.97 \\
8 & 65207.50 & 65207.39 & 29 & & 66797.48 \\
9 & 65569.06 & 65568.98 & 30 & & 66806.04 \\
10 & 65827.38 & 65827.52 & 35 & & 66838.44 \\
11 & 66018.75 & 66018.75 & 40 & & 66859.46 \\
12 & & 66164.14 & 45 & & 66873.87 \\
13 & & 66277.27 & 50 & & 66884.18 \\
14 & & 66367.01 & 55 & & 66891.80 \\
15 & & 66439.40 & 60 & & 66897.60 \\
16 & & 66498.63 & 65 & & 66902.11 \\
17 & & 66547.71 & 70 & & 66905.69 \\
18 & & 66588.83 & 75 & & 66908.58 \\
19 & & 66623.63 & 80 & & 66910.95 \\
20 & & 66653.34 & 85 & & 66912.91 \\
21 & & 66678.90 & 90 & & 66914.55 \\
22 & & 66701.05 & 95 & & 66915.94 \\
23 & & 66720.38 & 100 & & 66917.12 \\
24 & & 66737.34 & & &
\end{tabular}

TABLE V

Calculated results of energy levels $\left(\mathrm{cm}^{-1}\right)$ for BI $1 s^{2} 2 s^{2} n f{ }^{2} F_{7 / 2}$ (limit is $66928.10 \mathrm{~cm}^{-1}$ ) series compared with experimental data $\left(\mathrm{cm}^{-1}\right)$.

\begin{tabular}{|c|c|c|c|c|c|}
\hline$n$ & $T_{\exp }[48]$ & $T_{\text {cal }}$ & $n$ & $T_{\exp }[48]$ & $T_{\text {cal }}$ \\
\hline 4 & 60031.03 & 60031.03 & 25 & & 66752.30 \\
\hline 5 & 62516.52 & 62516.52 & 26 & & 66765.57 \\
\hline 6 & 63866.33 & 63866.33 & 27 & & 66777.40 \\
\hline 7 & 64679.74 & 64679.74 & 28 & & 66787.97 \\
\hline 8 & 65207.50 & 65207.39 & 29 & & 66797.48 \\
\hline 9 & 65569.06 & 65568.98 & 30 & & 66806.04 \\
\hline 10 & 65827.38 & 65827.52 & 35 & & 66838.44 \\
\hline 11 & & 66018.75 & 40 & & 66859.46 \\
\hline 12 & & 66164.14 & 45 & & 66873.87 \\
\hline 13 & & 66277.27 & 50 & & 66884.18 \\
\hline 14 & & 66367.01 & 55 & & 66891.80 \\
\hline 15 & & 66439.40 & 60 & & 66897.60 \\
\hline 16 & & 66498.63 & 65 & & 66902.11 \\
\hline 17 & & 66547.71 & 70 & & 66905.69 \\
\hline 18 & & 66588.83 & 75 & & 66908.58 \\
\hline 19 & & 66623.63 & 80 & & 66910.95 \\
\hline 20 & & 66653.34 & 85 & & 66912.91 \\
\hline 21 & & 66678.90 & 90 & & 66914.55 \\
\hline 22 & & 66701.05 & 95 & & 66915.94 \\
\hline 23 & & 66720.38 & 100 & & 66917.12 \\
\hline 24 & & 66737.34 & & & \\
\hline
\end{tabular}

\section{TABLE VI}

Parameters $a$ and $b$ of every tables.

\begin{tabular}{|c|c|c|c|c|c|c|c|c|}
\hline & $a_{1}$ & $a_{2}$ & $a_{3}$ & $a_{4}$ & $b_{1}$ & $b_{2}$ & $b_{3}$ & $b_{4}$ \\
\hline Table $\mathrm{I}^{a}$ & 1.24532 & -7.61248 & 61.96560 & -144.14765 & & & & \\
\hline Table $\mathrm{I}^{b}$ & 0.93534 & 0.51214 & -4.16833 & 11.27022 & 0.00074 & & & \\
\hline Table II & 0.04127 & -0.26264 & 0.01118 & -11.98405 & & & & \\
\hline Table III & 0.04207 & -0.31054 & 0.81200 & -15.91133 & & & & \\
\hline Table IV & 0.01563 & -0.11462 & 1.37123 & -10.70191 & & & & \\
\hline Table V & 0.01563 & -0.11462 & 1.37123 & -10.70191 & & & & \\
\hline
\end{tabular}

${ }^{a}$ without perturbation; ${ }^{b}$ with perturbation

However, the transitions probabilities for individual line have many applications in the corresponding fields, so present calculation is very significant. Good agreement is obtained from the comparison in this paper. We predict many values in the case of the NIST values not existing, the good agreement enables us to believe the predicted results are reliable.

Because most theoretical methods are only used to obtain transition probabilities for multiplets, in order to make the comparison with the other method and test the reliability of our method further, we also calculate transition probabilities and oscillator strengths BI for multi- plets. The results are listed in Table VIII. In Table VIII the transition rates performed using the MCHF method [10] are listed in the fifth column and the oscillator strength calculated by other method are given in the sixth column. From the comparison our results are generally closer to the accepted values than the others and in the case of the accepted values do not exist, our results are close to the others.

In conclusion, the WBEPM theory is successful in studying the energy levels and transition probabilities for BI. The calculation procedure is simple and the results are accurate. 
TABLE VII

Transition probabilities and oscillator strengths for individual lines of BI and comparison with accepted values.

\begin{tabular}{|c|c|c|c|c|c|}
\hline Transition & $\begin{array}{c}\text { Present transition } \\
\text { probabilities } \\
{\left[10^{8} \mathrm{~s}^{-1}\right]}\end{array}$ & $\begin{array}{c}\text { NIST transition } \\
\text { probabilities } \\
{\left[10^{8} \mathrm{~s}^{-1}\right][48]}\end{array}$ & \begin{tabular}{|c|} 
Present \\
oscillator \\
strengths
\end{tabular} & $\begin{array}{c}\text { NIST oscillator } \\
\text { strengths } \\
{[48]}\end{array}$ & $\begin{array}{c}\text { Accuraccy } \\
\text { of NIST } \\
{[48]}\end{array}$ \\
\hline $3 s{ }^{2} S_{1 / 2}-3 p{ }^{2} P_{1 / 2}^{0}$ & $1.73 \times 10^{-1}$ & 0.174 & $3.53 \times 10^{-1}$ & 0.355 & $\mathrm{C}$ \\
\hline $3 s{ }^{2} S_{1 / 2}-3 p{ }^{2} P_{3 / 2}^{0}$ & $1.73 \times 10^{-1}$ & 0.174 & $7.07 \times 10^{-1}$ & 0.710 & $\mathrm{C}$ \\
\hline $3 s{ }^{2} S_{1 / 2}-4 p{ }^{2} P_{1 / 2}^{0}$ & $3.84 \times 10^{-3}$ & & $1.83 \times 10^{-3}$ & & \\
\hline $3 s{ }^{2} S_{1 / 2}-4 p^{2} P_{3 / 2}^{0}$ & $3.85 \times 10^{-3}$ & & $3.67 \times 10^{-3}$ & & \\
\hline $4 s^{2} S_{1 / 2}-3 p{ }^{2} P_{1 / 2}^{0}$ & $4.91 \times 10^{-2}$ & 0.051 & $1.80 \times 10^{-1}$ & 0.19 & $\mathrm{C}$ \\
\hline $4 s^{2} S_{1 / 2}-3 p{ }^{2} P_{3 / 2}^{0}$ & $9.83 \times 10^{-2}$ & 0.103 & $1.80 \times 10^{-1}$ & 0.189 & $\mathrm{C}$ \\
\hline $4 s^{2} S_{1 / 2}-4 p{ }^{2} P_{1 / 2}^{0}$ & $2.65 \times 10^{-2}$ & & $5.16 \times 10^{-1}$ & & \\
\hline $4 s^{2} S_{1 / 2}-4 p^{2} P_{3 / 2}^{0}$ & $2.66 \times 10^{-2}$ & & 1.03 & & \\
\hline $5 s{ }^{2} S_{1 / 2}-3 p{ }^{2} P_{1 / 2}^{0}$ & $1.93 \times 10^{-2}$ & 0.0162 & $2.17 \times 10^{-2}$ & 0.0183 & $\mathrm{C}$ \\
\hline $5 s{ }^{2} S_{1 / 2}-3 p{ }^{2} P_{3 / 2}^{0}$ & $3.85 \times 10^{-2}$ & 0.0324 & $2.17 \times 10^{-2}$ & 0.0183 & $\mathrm{C}$ \\
\hline $5 s{ }^{2} S_{1 / 2}-4 p{ }^{2} P_{1 / 2}^{0}$ & $1.21 \times 10^{-2}$ & & $3.25 \times 10^{-1}$ & & \\
\hline $5 s^{2} S_{1 / 2}-4 p^{2} P_{3 / 2}^{0}$ & $2.42 \times 10^{-2}$ & & $3.26 \times 10^{-1}$ & & \\
\hline $6 s^{2} S_{1 / 2}-3 p{ }^{2} P_{1 / 2}^{0}$ & $8.76 \times 10^{-3}$ & & $6.82 \times 10^{-3}$ & & \\
\hline $6 s{ }^{2} S_{1 / 2}-3 p{ }^{2} P_{3 / 2}^{0}$ & $1.75 \times 10^{-2}$ & & $6.82 \times 10^{-3}$ & & \\
\hline $6 s{ }^{2} S_{1 / 2}-4 p{ }^{2} P_{1 / 2}^{0}$ & $4.33 \times 10^{-3}$ & & $2.94 \times 10^{-2}$ & & \\
\hline $6 s{ }^{2} S_{1 / 2}-4 p{ }^{2} P_{3 / 2}^{0}$ & $8.65 \times 10^{-3}$ & & $2.94 \times 10^{-2}$ & & \\
\hline $7 s{ }^{2} S_{1 / 2}-3 p{ }^{2} P_{1 / 2}^{0}$ & $3.93 \times 10^{-3}$ & & $2.44 \times 10^{-3}$ & & \\
\hline $7 s{ }^{2} S_{1 / 2}-3 p{ }^{2} P_{3 / 2}^{0}$ & $7.87 \times 10^{-3}$ & & $2.44 \times 10^{-3}$ & & \\
\hline $7 s^{2} S_{1 / 2}-4 p^{2} P_{1 / 2}^{0}$ & $2.60 \times 10^{-3}$ & & $9.62 \times 10^{-3}$ & & \\
\hline $7 s^{2} S_{1 / 2}-4 p^{2} P_{3 / 2}^{0}$ & $5.21 \times 10^{-3}$ & & $9.62 \times 10^{-3}$ & & \\
\hline $8 s{ }^{2} S_{1 / 2}-3 p{ }^{2} P_{1 / 2}^{0}$ & $3.54 \times 10^{-3}$ & & $2.03 \times 10^{-3}$ & & \\
\hline $8 s{ }^{2} S_{1 / 2}-3 p{ }^{2} P_{3 / 2}^{0}$ & $7.08 \times 10^{-3}$ & & $2.03 \times 10^{-3}$ & & \\
\hline $8 s^{2} S_{1 / 2}-4 p^{2} P_{1 / 2}^{0}$ & $2.02 \times 10^{-3}$ & & $6.16 \times 10^{-3}$ & & \\
\hline $8 s{ }^{2} S_{1 / 2}-4 p{ }^{2} P_{3 / 2}^{0}$ & $4.03 \times 10^{-3}$ & & $6.16 \times 10^{-3}$ & & \\
\hline $9 s^{2} S_{1 / 2}-3 p{ }^{2} P_{1 / 2}^{0}$ & $2.47 \times 10^{-3}$ & & $1.33 \times 10^{-3}$ & & \\
\hline $9 s{ }^{2} S_{1 / 2}-3 p{ }^{2} P_{3 / 2}^{0}$ & $4.94 \times 10^{-3}$ & & $1.33 \times 10^{-3}$ & & \\
\hline $9 s^{2} S_{1 / 2}-4 p^{2} P_{1 / 2}^{0}$ & $1.35 \times 10^{-3}$ & & $3.61 \times 10^{-3}$ & & \\
\hline $9 s{ }^{2} S_{1 / 2}-4 p^{2} P_{3 / 2}^{0}$ & $2.70 \times 10^{-3}$ & & $3.61 \times 10^{-3}$ & & \\
\hline $10 s{ }^{2} S_{1 / 2}-3 p{ }^{2} P_{1 / 2}^{0}$ & $1.76 \times 10^{-3}$ & & $9.11 \times 10^{-4}$ & & \\
\hline $10 s{ }^{2} S_{1 / 2}-3 p{ }^{2} P_{3 / 2}^{0}$ & $3.51 \times 10^{-3}$ & & $9.11 \times 10^{-4}$ & & \\
\hline $10 s^{2} S_{1 / 2}-4 p{ }^{2} P_{1 / 2}^{0}$ & $9.39 \times 10^{-4}$ & & $2.30 \times 10^{-3}$ & & \\
\hline $10 s^{2} S_{1 / 2}-4 p{ }^{2} P_{3 / 2}^{0}$ & $1.88 \times 10^{-3}$ & & $2.30 \times 10^{-3}$ & & \\
\hline $3 p^{2} P_{1 / 2}^{0}-3 d^{2} D_{3 / 2}$ & $1.13 \times 10^{-1}$ & 0.115 & $8.96 \times 10^{-1}$ & 0.91 & $\mathrm{C}$ \\
\hline $3 p^{2} P_{1 / 2}^{0}-4 d^{2} D_{3 / 2}$ & $8.66 \times 10^{-4}$ & & $2.00 \times 10^{-3}$ & & \\
\hline $3 p^{2} P_{1 / 2}^{0}-5 d^{2} D_{3 / 2}$ & $3.13 \times 10^{-4}$ & & $4.87 \times 10^{-4}$ & & \\
\hline $3 p^{2} P_{1 / 2}^{0}-6 d^{2} D_{3 / 2}$ & $6.88 \times 10^{-4}$ & & $8.89 \times 10^{-4}$ & & \\
\hline $3 p^{2} P_{1 / 2}^{0}-7 d^{2} D_{3 / 2}$ & $6.91 \times 10^{-4}$ & & $8.03 \times 10^{-4}$ & & \\
\hline $3 p^{2} P_{1 / 2}^{0}-8 d^{2} D_{3 / 2}$ & $5.82 \times 10^{-4}$ & & $6.35 \times 10^{-4}$ & & \\
\hline $3 p^{2} P_{1 / 2}^{0}-9 d^{2} D_{3 / 2}$ & $4.70 \times 10^{-4}$ & & $4.91 \times 10^{-4}$ & & \\
\hline $3 p^{2} P_{1 / 2}^{0}-10 d^{2} D_{3 / 2}$ & $3.73 \times 10^{-4}$ & & $3.77 \times 10^{-4}$ & & \\
\hline $3 p^{2} P_{3 / 2}^{0}-3 d^{2} D_{3 / 2}$ & $2.26 \times 10^{-2}$ & 0.023 & $8.96 \times 10^{-2}$ & 0.091 & $\mathrm{C}$ \\
\hline $3 p^{2} P_{3 / 2}^{0}-3 d^{2} D_{5 / 2}$ & $1.36 \times 10^{-1}$ & 0.138 & $8.06 \times 10^{-1}$ & 0.819 & $\mathrm{C}$ \\
\hline
\end{tabular}


TABLE VII (cont.)

TABLE VII (cont.)

\begin{tabular}{|c|c|c|}
\hline Transition & $\begin{array}{c}\text { Present transition } \\
\text { probabilities }\left[10^{8} \mathrm{~s}^{-1}\right]\end{array}$ & $\begin{array}{l}\text { Present oscillator } \\
\text { strengths }\end{array}$ \\
\hline $3 p^{2} P_{3 / 2}^{0}-4 d^{2} D_{3 / 2}$ & $1.72 \times 10^{-4}$ & $1.99 \times 10^{-4}$ \\
\hline $3 p^{2} P_{3 / 2}^{0}-4 d^{2} D_{5 / 2}$ & $1.03 \times 10^{-3}$ & $1.79 \times 10^{-3}$ \\
\hline $3 p^{2} P_{3 / 2}^{0}-5 d^{2} D_{3 / 2}$ & $6.31 \times 10^{-5}$ & $4.91 \times 10^{-5}$ \\
\hline $3 p^{2} P_{3 / 2}^{0}-5 d^{2} D_{5 / 2}$ & $3.76 \times 10^{-4}$ & $4.40 \times 10^{-4}$ \\
\hline $3 p^{2} P_{3 / 2}^{0}-6 d^{2} D_{3 / 2}$ & $1.38 \times 10^{-4}$ & $8.93 \times 10^{-5}$ \\
\hline $3 p^{2} P_{3 / 2}^{0}-6 d^{2} D_{5 / 2}$ & $8.29 \times 10^{-4}$ & $8.04 \times 10^{-4}$ \\
\hline $3 p^{2} P_{3 / 2}^{0}-7 d^{2} D_{3 / 2}$ & $1.39 \times 10^{-4}$ & $8.06 \times 10^{-5}$ \\
\hline $3 p^{2} P_{3 / 2}^{0}-7 d^{2} D_{5 / 2}$ & $8.30 \times 10^{-4}$ & $7.25 \times 10^{-4}$ \\
\hline $3 p^{2} P_{3 / 2}^{0}-8 d^{2} D_{3 / 2}$ & $1.17 \times 10^{-4}$ & $6.37 \times 10^{-5}$ \\
\hline $3 p^{2} P_{3 / 2}^{0}-8 d^{2} D_{5 / 2}$ & $7.02 \times 10^{-4}$ & $5.74 \times 10^{-4}$ \\
\hline $3 p^{2} P_{3 / 2}^{0}-9 d^{2} D_{3 / 2}$ & $9.43 \times 10^{-5}$ & $4.92 \times 10^{-5}$ \\
\hline $3 p^{2} P_{3 / 2}^{0}-9 d^{2} D_{5 / 2}$ & $5.66 \times 10^{-4}$ & $4.43 \times 10^{-4}$ \\
\hline $3 p^{2} P_{3 / 2}^{0}-10 d^{2} D_{3 / 2}$ & $7.48 \times 10^{-5}$ & $3.78 \times 10^{-5}$ \\
\hline $3 p^{2} P_{3 / 2}^{0}-10 d^{2} D_{5 / 2}$ & $4.49 \times 10^{-4}$ & $3.41 \times 10^{-4}$ \\
\hline $4 p^{2} P_{1 / 2}^{0}-3 d^{2} D_{3 / 2}$ & $1.80 \times 10^{-2}$ & $1.48 \times 10^{-1}$ \\
\hline $4 p^{2} P_{1 / 2}^{0}-4 d^{2} D_{3 / 2}$ & $2.04 \times 10^{-2}$ & 1.26 \\
\hline $4 p^{2} P_{1 / 2}^{0}-5 d^{2} D_{3 / 2}$ & $1.19 \times 10^{-3}$ & $1.62 \times 10^{-2}$ \\
\hline $4 p^{2} P_{1 / 2}^{0}-6 d^{2} D_{3 / 2}$ & $9.92 \times 10^{-5}$ & $8.10 \times 10^{-4}$ \\
\hline $4 p^{2} P_{1 / 2}^{0}-7 d^{2} D_{3 / 2}$ & $2.50 \times 10^{-6}$ & $1.58 \times 10^{-5}$ \\
\hline $4 p^{2} P_{1 / 2}^{0}-8 d^{2} D_{3 / 2}$ & $2.68 \times 10^{-6}$ & $1.46 \times 10^{-5}$ \\
\hline $4 p^{2} P_{1 / 2}^{0}-9 d^{2} D_{3 / 2}$ & $8.74 \times 10^{-6}$ & $4.34 \times 10^{-5}$ \\
\hline $4 p^{2} P_{1 / 2}^{0}-10 d{ }^{2} D_{3 / 2}$ & $1.15 \times 10^{-5}$ & $5.33 \times 10^{-5}$ \\
\hline $4 p^{2} P_{3 / 2}^{0}-3 d^{2} D_{3 / 2}$ & $1.80 \times 10^{-3}$ & $2.97 \times 10^{-2}$ \\
\hline $4 p^{2} P_{3 / 2}^{0}-3 d^{2} D_{5 / 2}$ & $1.62 \times 10^{-2}$ & $1.78 \times 10^{-1}$ \\
\hline $4 p^{2} P_{3 / 2}^{0}-4 d^{2} D_{3 / 2}$ & $4.08 \times 10^{-3}$ & $1.26 \times 10^{-1}$ \\
\hline $4 p^{2} P_{3 / 2}^{0}-4 d^{2} D_{5 / 2}$ & $2.45 \times 10^{-2}$ & 1.13 \\
\hline $4 p^{2} P_{3 / 2}^{0}-5 d^{2} D_{3 / 2}$ & $2.38 \times 10^{-4}$ & $1.62 \times 10^{-3}$ \\
\hline $4 p^{2} P_{3 / 2}^{0}-5 d^{2} D_{5 / 2}$ & $1.43 \times 10^{-3}$ & $1.46 \times 10^{-2}$ \\
\hline $4 p^{2} P_{3 / 2}^{0}-6 d^{2} D_{3 / 2}$ & $1.97 \times 10^{-5}$ & $8.04 \times 10^{-5}$ \\
\hline $4 p^{2} P_{3 / 2}^{0}-6 d^{2} D_{5 / 2}$ & $1.18 \times 10^{-4}$ & $7.23 \times 10^{-4}$ \\
\hline $4 p^{2} P_{3 / 2}^{0}-7 d^{2} D_{3 / 2}$ & $4.80 \times 10^{-7}$ & $1.52 \times 10^{-6}$ \\
\hline $4 p^{2} P_{3 / 2}^{0}-7 d^{2} D_{5 / 2}$ & $2.94 \times 10^{-6}$ & $1.40 \times 10^{-5}$ \\
\hline $4 p^{2} P_{3 / 2}^{0}-8 d^{2} D_{3 / 2}$ & $5.52 \times 10^{-7}$ & $1.51 \times 10^{-6}$ \\
\hline $4 p^{2} P_{3 / 2}^{0}-8 d^{2} D_{5 / 2}$ & $3.35 \times 10^{-6}$ & $1.37 \times 10^{-5}$ \\
\hline $4 p^{2} P_{3 / 2}^{0}-9 d^{2} D_{3 / 2}$ & $1.77 \times 10^{-6}$ & $4.40 \times 10^{-6}$ \\
\hline $4 p^{2} P_{3 / 2}^{0}-9 d^{2} D_{5 / 2}$ & $1.06 \times 10^{-5}$ & $3.96 \times 10^{-5}$ \\
\hline $4 p^{2} P_{3 / 2}^{0}-10 d^{2} D_{3 / 2}$ & $2.32 \times 10^{-6}$ & $5.39 \times 10^{-6}$ \\
\hline $4 p^{2} P_{3 / 2}^{0}-10 d^{2} D_{5 / 2}$ & $1.39 \times 10^{-5}$ & $4.85 \times 10^{-5}$ \\
\hline $3 d^{2} D_{3 / 2}-4 f^{2} F_{5 / 2}^{0}$ & $1.26 \times 10^{-1}$ & 1.02 \\
\hline $3 d^{2} D_{3 / 2}-5 f^{2} F_{5 / 2}^{0}$ & $3.98 \times 10^{-2}$ & $1.49 \times 10^{-1}$ \\
\hline $3 d^{2} D_{3 / 2}-6 f^{2} F_{5 / 2}^{0}$ & $1.84 \times 10^{-2}$ & $5.01 \times 10^{-2}$ \\
\hline $3 d^{2} D_{3 / 2}-7 f^{2} F_{5 / 2}^{0}$ & $1.03 \times 10^{-2}$ & $2.35 \times 10^{-2}$ \\
\hline $3 d^{2} D_{3 / 2}-8 f^{2} F_{5 / 2}^{0}$ & $6.39 \times 10^{-3}$ & $1.32 \times 10^{-2}$ \\
\hline $3 d^{2} D_{3 / 2}-9 f^{2} F_{5 / 2}^{0}$ & $4.27 \times 10^{-3}$ & $8.23 \times 10^{-3}$ \\
\hline $3 d^{2} D_{3 / 2}-10 f^{2} F_{5 / 2}^{0}$ & $2.99 \times 10^{-3}$ & $5.50 \times 10^{-3}$ \\
\hline $3 d^{2} D_{3 / 2}-11 f^{2} F_{5 / 2}^{0}$ & $2.20 \times 10^{-3}$ & $3.91 \times 10^{-3}$ \\
\hline $3 d^{2} D_{5 / 2}-4 f^{2} F_{5 / 2}^{0}$ & $9.02 \times 10^{-3}$ & $4.88 \times 10^{-2}$ \\
\hline $3 d^{2} D_{5 / 2}-4 f^{2} F_{7 / 2}^{0}$ & $1.35 \times 10^{-1}$ & $9.76 \times 10^{-1}$ \\
\hline $3 d^{2} D_{5 / 2}-5 f^{2} F_{5 / 2}^{0}$ & $2.84 \times 10^{-3}$ & $7.09 \times 10^{-3}$ \\
\hline $3 d^{2} D_{5 / 2}-5 f^{2} F_{7 / 2}^{0}$ & $4.26 \times 10^{-2}$ & $1.42 \times 10^{-1}$ \\
\hline $3 d^{2} D_{5 / 2}-6 f^{2} F_{5 / 2}^{0}$ & $1.32 \times 10^{-3}$ & $2.39 \times 10^{-3}$ \\
\hline $3 d^{2} D_{5 / 2}-6 f^{2} F_{7 / 2}^{0}$ & $1.98 \times 10^{-2}$ & $4.77 \times 10^{-2}$ \\
\hline $3 d^{2} D_{5 / 2}-7 f^{2} F_{5 / 2}^{0}$ & $7.34 \times 10^{-4}$ & $1.12 \times 10^{-3}$ \\
\hline
\end{tabular}

\begin{tabular}{|c|c|c|}
\hline Transition & $\begin{array}{c}\text { Present transition } \\
\text { probabilities }\left[10^{8} \mathrm{~s}^{-1}\right]\end{array}$ & $\begin{array}{l}\text { Present oscillator } \\
\text { strengths }\end{array}$ \\
\hline $3 d^{2} D_{5 / 2}-7 f^{2} F_{7 / 2}^{0}$ & $1.10 \times 10^{-2}$ & $2.24 \times 10^{-2}$ \\
\hline $3 d^{2} D_{5 / 2}-8 f^{2} F_{5 / 2}^{0}$ & $4.56 \times 10^{-4}$ & $6.28 \times 10^{-4}$ \\
\hline $3 d^{2} D_{5 / 2}-8 f^{2} F_{7 / 2}^{0}$ & $6.85 \times 10^{-3}$ & $1.26 \times 10^{-2}$ \\
\hline $3 d^{2} D_{5 / 2}-9 f^{2} F_{5 / 2}^{0}$ & $3.05 \times 10^{-4}$ & $3.92 \times 10^{-4}$ \\
\hline $3 d^{2} D_{5 / 2}-9 f^{2} F_{7 / 2}^{0}$ & $4.57 \times 10^{-3}$ & $7.83 \times 10^{-3}$ \\
\hline $3 d^{2} D_{5 / 2}-10 f^{2} F_{5 / 2}^{0}$ & $2.14 \times 10^{-4}$ & $2.62 \times 10^{-4}$ \\
\hline $3 d^{2} D_{5 / 2}-10 f^{2} F_{7 / 2}^{0}$ & $3.20 \times 10^{-3}$ & $5.24 \times 10^{-3}$ \\
\hline $3 d^{2} D_{5 / 2}-11 f^{2} F_{5 / 2}^{0}$ & $1.57 \times 10^{-4}$ & $1.86 \times 10^{-4}$ \\
\hline $4 d^{2} D_{3 / 2}-4 f^{2} F_{5 / 2}^{0}$ & $1.08 \times 10^{-7}$ & $1.72 \times 10^{-2}$ \\
\hline $4 d^{2} D_{3 / 2}-5 f{ }^{2} F_{5 / 2}^{0}$ & $2.44 \times 10^{-2}$ & $8.61 \times 10^{-1}$ \\
\hline $4 d^{2} D_{3 / 2}-6 f^{2} F_{5 / 2}^{0}$ & $1.23 \times 10^{-2}$ & $1.85 \times 10^{-1}$ \\
\hline $4 d^{2} D_{3 / 2}-7 f^{2} F_{5 / 2}^{0}$ & $7.07 \times 10^{-3}$ & $7.24 \times 10^{-2}$ \\
\hline $4 d^{2} D_{3 / 2}-8 f^{2} F_{5 / 2}^{0}$ & $4.46 \times 10^{-3}$ & $3.69 \times 10^{-2}$ \\
\hline $4 d^{2} D_{3 / 2}-9 f^{2} F_{5 / 2}^{0}$ & $3.01 \times 10^{-3}$ & $2.17 \times 10^{-2}$ \\
\hline $4 d^{2} D_{3 / 2}-10 f^{2} F_{5 / 2}^{0}$ & $2.12 \times 10^{-3}$ & $1.40 \times 10^{-2}$ \\
\hline $4 d^{2} D_{3 / 2}-11 f^{2} F_{5 / 2}^{0}$ & $1.56 \times 10^{-3}$ & $9.68 \times 10^{-3}$ \\
\hline $4 d^{2} D_{5 / 2}-4 f^{2} F_{5 / 2}^{0}$ & $7.68 \times 10^{-9}$ & $8.18 \times 10^{-4}$ \\
\hline $4 d^{2} D_{5 / 2}-4 f^{2} F_{7 / 2}^{0}$ & $1.15 \times 10^{-7}$ & $1.64 \times 10^{-2}$ \\
\hline $4 d^{2} D_{5 / 2}-5 f^{2} F_{5 / 2}^{0}$ & $1.74 \times 10^{-3}$ & $4.10 \times 10^{-2}$ \\
\hline $4 d^{2} D_{5 / 2}-5 f^{2} F_{7 / 2}^{0}$ & $2.61 \times 10^{-2}$ & $8.20 \times 10^{-1}$ \\
\hline $4 d^{2} D_{5 / 2}-6 f^{2} F_{5 / 2}^{0}$ & $8.80 \times 10^{-4}$ & $8.80 \times 10^{-3}$ \\
\hline $4 d^{2} D_{5 / 2}-6 f^{2} F_{7 / 2}^{0}$ & $1.32 \times 10^{-2}$ & $1.76 \times 10^{-1}$ \\
\hline $4 d^{2} D_{5 / 2}-7 f^{2} F_{5 / 2}^{0}$ & $5.05 \times 10^{-4}$ & $3.45 \times 10^{-3}$ \\
\hline $4 d^{2} D_{5 / 2}-7 f^{2} F_{7 / 2}^{0}$ & $7.57 \times 10^{-3}$ & $6.89 \times 10^{-2}$ \\
\hline $4 d^{2} D_{5 / 2}-8 f^{2} F_{5 / 2}^{0}$ & $3.18 \times 10^{-4}$ & $1.76 \times 10^{-3}$ \\
\hline $4 d^{2} D_{5 / 2}-8 f^{2} F_{7 / 2}^{0}$ & $4.78 \times 10^{-3}$ & $3.51 \times 10^{-2}$ \\
\hline $4 d^{2} D_{5 / 2}-9 f^{2} F_{5 / 2}^{0}$ & $2.15 \times 10^{-4}$ & $1.04 \times 10^{-3}$ \\
\hline $4 d^{2} D_{5 / 2}-9 f^{2} F_{7 / 2}^{0}$ & $3.22 \times 10^{-3}$ & $2.07 \times 10^{-2}$ \\
\hline $4 d^{2} D_{5 / 2}-10 f^{2} F_{5 / 2}^{0}$ & $1.51 \times 10^{-4}$ & $6.67 \times 10^{-4}$ \\
\hline $4 d^{2} D_{5 / 2}-10 f^{2} F_{7 / 2}^{0}$ & $2.27 \times 10^{-3}$ & $1.33 \times 10^{-2}$ \\
\hline $4 d^{2} D_{5 / 2}-11 f^{2} F_{5 / 2}^{0}$ & $1.12 \times 10^{-4}$ & $4.61 \times 10^{-4}$ \\
\hline $5 d^{2} D_{3 / 2}-4 f^{2} F_{5 / 2}^{0}$ & $6.37 \times 10^{-4}$ & $1.06 \times 10^{-2}$ \\
\hline $5 d^{2} D_{3 / 2}-5 f^{2} F_{5 / 2}^{0}$ & $2.19 \times 10^{-7}$ & $5.10 \times 10^{-2}$ \\
\hline $5 d^{2} D_{3 / 2}-6 f^{2} F_{5 / 2}^{0}$ & $6.68 \times 10^{-3}$ & $7.87 \times 10^{-1}$ \\
\hline $5 d^{2} D_{3 / 2}-7 f^{2} F_{5 / 2}^{0}$ & $4.11 \times 10^{-3}$ & $1.92 \times 10^{-1}$ \\
\hline $5 d^{2} D_{3 / 2}-8 f^{2} F_{5 / 2}^{0}$ & $2.64 \times 10^{-3}$ & $8.02 \times 10^{-2}$ \\
\hline $5 d^{2} D_{3 / 2}-9 f^{2} F_{5 / 2}^{0}$ & $1.80 \times 10^{-3}$ & $4.25 \times 10^{-2}$ \\
\hline $5 d^{2} D_{3 / 2}-10 f^{2} F_{5 / 2}^{0}$ & $1.28 \times 10^{-3}$ & $2.57 \times 10^{-2}$ \\
\hline $5 d^{2} D_{3 / 2}-11 f^{2} F_{5 / 2}^{0}$ & $9.43 \times 10^{-4}$ & $1.70 \times 10^{-2}$ \\
\hline $5 d^{2} D_{5 / 2}-4 f^{2} F_{5 / 2}^{0}$ & $3.03 \times 10^{-5}$ & $7.53 \times 10^{-4}$ \\
\hline $5 d^{2} D_{5 / 2}-4 f^{2} F_{7 / 2}^{0}$ & $6.06 \times 10^{-4}$ & $1.13 \times 10^{-2}$ \\
\hline $5 d^{2} D_{5 / 2}-5 f^{2} F_{5 / 2}^{0}$ & $1.54 \times 10^{-8}$ & $2.42 \times 10^{-3}$ \\
\hline $5 d^{2} D_{5 / 2}-5 f^{2} F_{7 / 2}^{0}$ & $2.31 \times 10^{-7}$ & $4.83 \times 10^{-2}$ \\
\hline $5 d^{2} D_{5 / 2}-6 f^{2} F_{5 / 2}^{0}$ & $4.77 \times 10^{-4}$ & $3.75 \times 10^{-2}$ \\
\hline $5 d^{2} D_{5 / 2}-6 f^{2} F_{7 / 2}^{0}$ & $7.15 \times 10^{-3}$ & $7.50 \times 10^{-1}$ \\
\hline $5 d^{2} D_{5 / 2}-7 f^{2} F_{5 / 2}^{0}$ & $2.94 \times 10^{-4}$ & $9.14 \times 10^{-3}$ \\
\hline $5 d^{2} D_{5 / 2}-7 f^{2} F_{7 / 2}^{0}$ & $4.40 \times 10^{-3}$ & $1.83 \times 10^{-1}$ \\
\hline $5 d^{2} D_{5 / 2}-8 f^{2} F_{5 / 2}^{0}$ & $1.89 \times 10^{-4}$ & $3.82 \times 10^{-3}$ \\
\hline $5 d^{2} D_{5 / 2}-8 f^{2} F_{7 / 2}^{0}$ & $2.83 \times 10^{-3}$ & $7.64 \times 10^{-2}$ \\
\hline $5 d^{2} D_{5 / 2}-9 f^{2} F_{5 / 2}^{0}$ & $1.28 \times 10^{-4}$ & $2.03 \times 10^{-3}$ \\
\hline $5 d^{2} D_{5 / 2}-9 f^{2} F_{7 / 2}^{0}$ & $1.93 \times 10^{-3}$ & $4.05 \times 10^{-2}$ \\
\hline $5 d^{2} D_{5 / 2}-10 f^{2} F_{5 / 2}^{0}$ & $9.12 \times 10^{-5}$ & $1.23 \times 10^{-3}$ \\
\hline $5 d^{2} D_{5 / 2}-10 f^{2} F_{7 / 2}^{0}$ & $1.37 \times 10^{-3}$ & $2.45 \times 10^{-2}$ \\
\hline
\end{tabular}


TABLE VII (cont.)

TABLE VII (cont.)

\begin{tabular}{|c|c|c|}
\hline Transition & $\begin{array}{c}\text { Present transition } \\
\text { probabilities }\left[10^{8} \mathrm{~s}^{-1}\right]\end{array}$ & $\begin{array}{c}\text { Present oscillator } \\
\text { strengths }\end{array}$ \\
\hline $5 d^{2} D_{5 / 2}-11 f^{2} F_{5 / 2}^{0}$ & $6.74 \times 10^{-5}$ & $8.09 \times 10^{-4}$ \\
\hline $6 d^{2} D_{3 / 2}-4 f^{2} F_{5 / 2}^{0}$ & $2.76 \times 10^{-4}$ & $1.90 \times 10^{-3}$ \\
\hline $6 d^{2} D_{3 / 2}-5 f^{2} F_{5 / 2}^{0}$ & $4.92 \times 10^{-4}$ & $2.79 \times 10^{-2}$ \\
\hline $6 d^{2} D_{3 / 2}-6 f^{2} F_{5 / 2}^{0}$ & $1.65 \times 10^{-7}$ & $8.39 \times 10^{-2}$ \\
\hline $6 d^{2} D_{3 / 2}-7 f^{2} F_{5 / 2}^{0}$ & $2.35 \times 10^{-3}$ & $7.57 \times 10^{-1}$ \\
\hline $6 d^{2} D_{3 / 2}-8 f^{2} F_{5 / 2}^{0}$ & $1.61 \times 10^{-3}$ & $1.95 \times 10^{-1}$ \\
\hline $6 d^{2} D_{3 / 2}-9 f^{2} F_{5 / 2}^{0}$ & $1.11 \times 10^{-3}$ & $8.43 \times 10^{-2}$ \\
\hline $6 d^{2} D_{3 / 2}-10 f^{2} F_{5 / 2}^{0}$ & $7.98 \times 10^{-4}$ & $4.57 \times 10^{-2}$ \\
\hline $6 d^{2} D_{3 / 2}-11 f^{2} F_{5 / 2}^{0}$ & $5.91 \times 10^{-4}$ & $2.81 \times 10^{-2}$ \\
\hline $6 d^{2} D_{5 / 2}-4 f^{2} F_{5 / 2}^{0}$ & $1.32 \times 10^{-5}$ & $1.36 \times 10^{-4}$ \\
\hline $6 d^{2} D_{5 / 2}-4 f^{2} F_{7 / 2}^{0}$ & $2.63 \times 10^{-4}$ & $2.03 \times 10^{-3}$ \\
\hline $6 d^{2} D_{5 / 2}-5 f^{2} F_{5 / 2}^{0}$ & $2.34 \times 10^{-5}$ & $1.99 \times 10^{-3}$ \\
\hline $6 d^{2} D_{5 / 2}-5 f^{2} F_{7 / 2}^{0}$ & $4.69 \times 10^{-4}$ & $2.99 \times 10^{-2}$ \\
\hline $6 d^{2} D_{5 / 2}-6 f^{2} F_{5 / 2}^{0}$ & $1.18 \times 10^{-8}$ & $4.00 \times 10^{-3}$ \\
\hline $6 d^{2} D_{5 / 2}-6 f^{2} F_{7 / 2}^{0}$ & $1.77 \times 10^{-7}$ & $7.99 \times 10^{-2}$ \\
\hline $6 d^{2} D_{5 / 2}-7 f^{2} F_{5 / 2}^{0}$ & $1.67 \times 10^{-4}$ & $3.61 \times 10^{-2}$ \\
\hline $6 d^{2} D_{5 / 2}-7 f^{2} F_{7 / 2}^{0}$ & $2.51 \times 10^{-3}$ & $7.21 \times 10^{-1}$ \\
\hline $6 d^{2} D_{5 / 2}-8 f^{2} F_{5 / 2}^{0}$ & $1.15 \times 10^{-4}$ & $9.29 \times 10^{-3}$ \\
\hline $6 d^{2} D_{5 / 2}-8 f^{2} F_{7 / 2}^{0}$ & $1.73 \times 10^{-3}$ & $1.86 \times 10^{-1}$ \\
\hline $6 d^{2} D_{5 / 2}-9 f^{2} F_{5 / 2}^{0}$ & $7.96 \times 10^{-5}$ & $4.02 \times 10^{-3}$ \\
\hline $6 d^{2} D_{5 / 2}-9 f^{2} F_{7 / 2}^{0}$ & $1.19 \times 10^{-3}$ & $8.03 \times 10^{-2}$ \\
\hline $6 d^{2} D_{5 / 2}-10 f^{2} F_{5 / 2}^{0}$ & $5.70 \times 10^{-5}$ & $2.18 \times 10^{-3}$ \\
\hline $6 d^{2} D_{5 / 2}-10 f^{2} F_{7 / 2}^{0}$ & $8.55 \times 10^{-4}$ & $4.35 \times 10^{-2}$ \\
\hline $6 d^{2} D_{5 / 2}-11 f^{2} F_{5 / 2}^{0}$ & $4.22 \times 10^{-5}$ & $1.34 \times 10^{-3}$ \\
\hline $7 d^{2} D_{3 / 2}-4 f^{2} F_{5 / 2}^{0}$ & $1.47 \times 10^{-4}$ & $6.82 \times 10^{-4}$ \\
\hline $7 d^{2} D_{3 / 2}-5 f^{2} F_{5 / 2}^{0}$ & $2.42 \times 10^{-4}$ & $5.24 \times 10^{-3}$ \\
\hline $7 d^{2} D_{3 / 2}-6 f^{2} F_{5 / 2}^{0}$ & $3.17 \times 10^{-4}$ & $4.96 \times 10^{-2}$ \\
\hline $7 d^{2} D_{3 / 2}-7 f^{2} F_{5 / 2}^{0}$ & $1.08 \times 10^{-7}$ & $1.16 \times 10^{-1}$ \\
\hline $7 d^{2} D_{3 / 2}-8 f^{2} F_{5 / 2}^{0}$ & $9.77 \times 10^{-4}$ & $7.47 \times 10^{-1}$ \\
\hline $7 d^{2} D_{3 / 2}-9 f^{2} F_{5 / 2}^{0}$ & $7.20 \times 10^{-4}$ & $1.98 \times 10^{-1}$ \\
\hline $7 d^{2} D_{3 / 2}-10 f^{2} F_{5 / 2}^{0}$ & $5.24 \times 10^{-4}$ & $8.73 \times 10^{-2}$ \\
\hline $7 d^{2} D_{3 / 2}-11 f^{2} F_{5 / 2}^{0}$ & $3.90 \times 10^{-4}$ & $4.79 \times 10^{-2}$ \\
\hline $7 d^{2} D_{5 / 2}-4 f^{2} F_{5 / 2}^{0}$ & $6.98 \times 10^{-6}$ & $4.87 \times 10^{-5}$ \\
\hline $7 d^{2} D_{5 / 2}-4 f^{2} F_{7 / 2}^{0}$ & $1.40 \times 10^{-4}$ & $7.30 \times 10^{-4}$ \\
\hline $7 d^{2} D_{5 / 2}-5 f^{2} F_{5 / 2}^{0}$ & $1.15 \times 10^{-5}$ & $3.74 \times 10^{-4}$ \\
\hline $7 d^{2} D_{5 / 2}-5 f^{2} F_{7 / 2}^{0}$ & $2.30 \times 10^{-4}$ & $5.61 \times 10^{-3}$ \\
\hline $7 d^{2} D_{5 / 2}-6 f^{2} F_{5 / 2}^{0}$ & $1.51 \times 10^{-5}$ & $3.54 \times 10^{-3}$ \\
\hline $7 d^{2} D_{5 / 2}-6 f^{2} F_{7 / 2}^{0}$ & $3.02 \times 10^{-4}$ & $5.31 \times 10^{-2}$ \\
\hline $7 d^{2} D_{5 / 2}-7 f^{2} F_{5 / 2}^{0}$ & $7.63 \times 10^{-9}$ & $5.52 \times 10^{-3}$ \\
\hline $7 d^{2} D_{5 / 2}-7 f^{2} F_{7 / 2}^{0}$ & $1.14 \times 10^{-7}$ & $1.10 \times 10^{-1}$ \\
\hline $7 d^{2} D_{5 / 2}-8 f^{2} F_{5 / 2}^{0}$ & $6.98 \times 10^{-5}$ & $3.56 \times 10^{-2}$ \\
\hline $7 d^{2} D_{5 / 2}-8 f{ }^{2} F_{7 / 2}^{0}$ & $1.05 \times 10^{-3}$ & $7.12 \times 10^{-1}$ \\
\hline $7 d^{2} D_{5 / 2}-9 f^{2} F_{5 / 2}^{0}$ & $5.14 \times 10^{-5}$ & $9.44 \times 10^{-3}$ \\
\hline $7 d^{2} D_{5 / 2}-9 f^{2} F_{7 / 2}^{0}$ & $7.71 \times 10^{-4}$ & $1.89 \times 10^{-1}$ \\
\hline $7 d^{2} D_{5 / 2}-10 f^{2} F_{5 / 2}^{0}$ & $3.75 \times 10^{-5}$ & $4.16 \times 10^{-3}$ \\
\hline $7 d^{2} D_{5 / 2}-10 f^{2} F_{7 / 2}^{0}$ & $5.62 \times 10^{-4}$ & $8.32 \times 10^{-2}$ \\
\hline $7 d^{2} D_{5 / 2}-11 f^{2} F_{5 / 2}^{0}$ & $2.79 \times 10^{-5}$ & $2.28 \times 10^{-3}$ \\
\hline $8 d^{2} D_{3 / 2}-4 f^{2} F_{5 / 2}^{0}$ & $8.79 \times 10^{-5}$ & $3.29 \times 10^{-4}$ \\
\hline $8 d^{2} D_{3 / 2}-5 f^{2} F_{5 / 2}^{0}$ & $1.38 \times 10^{-4}$ & $1.92 \times 10^{-3}$ \\
\hline $8 d^{2} D_{3 / 2}-6 f^{2} F_{5 / 2}^{0}$ & $1.70 \times 10^{-4}$ & $9.60 \times 10^{-3}$ \\
\hline $8 d^{2} D_{3 / 2}-7 f^{2} F_{5 / 2}^{0}$ & $1.99 \times 10^{-4}$ & $7.41 \times 10^{-2}$ \\
\hline $8 d^{2} D_{3 / 2}-8 f^{2} F_{5 / 2}^{0}$ & $6.80 \times 10^{-8}$ & $1.47 \times 10^{-1}$ \\
\hline $8 d^{2} D_{3 / 2}-9 f^{2} F_{5 / 2}^{0}$ & $4.62 \times 10^{-4}$ & $7.51 \times 10^{-1}$ \\
\hline
\end{tabular}

\begin{tabular}{|c|c|c|}
\hline Transition & $\begin{array}{c}\text { Present transition } \\
\text { probabilities }\left[10^{8} \mathrm{~s}^{-1}\right]\end{array}$ & $\begin{array}{c}\text { Present oscillator } \\
\text { strengths }\end{array}$ \\
\hline $8 d^{2} D_{3 / 2}-10 f^{2} F_{5 / 2}^{0}$ & $3.58 \times 10^{-4}$ & $2.03 \times 10^{-1}$ \\
\hline $8 d^{2} D_{3 / 2}-11 f^{2} F_{5 / 2}^{0}$ & $2.70 \times 10^{-4}$ & $8.99 \times 10^{-2}$ \\
\hline $8 d^{2} D_{5 / 2}-4 f^{2} F_{5 / 2}^{0}$ & $4.19 \times 10^{-6}$ & $2.35 \times 10^{-5}$ \\
\hline $8 d^{2} D_{5 / 2}-4 f^{2} F_{7 / 2}^{0}$ & $8.37 \times 10^{-5}$ & $3.53 \times 10^{-4}$ \\
\hline $8 d^{2} D_{5 / 2}-5 f^{2} F_{5 / 2}^{0}$ & $6.58 \times 10^{-6}$ & $1.37 \times 10^{-4}$ \\
\hline $8 d^{2} D_{5 / 2}-5 f^{2} F_{7 / 2}^{0}$ & $1.32 \times 10^{-4}$ & $2.06 \times 10^{-3}$ \\
\hline $8 d^{2} D_{5 / 2}-6 f^{2} F_{5 / 2}^{0}$ & $8.10 \times 10^{-6}$ & $6.86 \times 10^{-4}$ \\
\hline $8 d^{2} D_{5 / 2}-6 f^{2} F_{7 / 2}^{0}$ & $1.62 \times 10^{-4}$ & $1.03 \times 10^{-2}$ \\
\hline $8 d^{2} D_{5 / 2}-7 f^{2} F_{5 / 2}^{0}$ & $9.47 \times 10^{-6}$ & $5.30 \times 10^{-3}$ \\
\hline $8 d^{2} D_{5 / 2}-7 f^{2} F_{7 / 2}^{0}$ & $1.89 \times 10^{-4}$ & $7.95 \times 10^{-2}$ \\
\hline $8 d^{2} D_{5 / 2}-8 f^{2} F_{5 / 2}^{0}$ & $4.88 \times 10^{-9}$ & $7.02 \times 10^{-3}$ \\
\hline $8 d^{2} D_{5 / 2}-8 f^{2} F_{7 / 2}^{0}$ & $7.33 \times 10^{-8}$ & $1.40 \times 10^{-1}$ \\
\hline $8 d^{2} D_{5 / 2}-9 f^{2} F_{5 / 2}^{0}$ & $3.30 \times 10^{-5}$ & $3.58 \times 10^{-2}$ \\
\hline $8 d^{2} D_{5 / 2}-9 f^{2} F_{7 / 2}^{0}$ & $4.95 \times 10^{-4}$ & $7.16 \times 10^{-1}$ \\
\hline $8 d^{2} D_{5 / 2}-10 f^{2} F_{5 / 2}^{0}$ & $2.55 \times 10^{-5}$ & $9.65 \times 10^{-3}$ \\
\hline $8 d^{2} D_{5 / 2}-10 f^{2} F_{7 / 2}^{0}$ & $3.83 \times 10^{-4}$ & $1.93 \times 10^{-1}$ \\
\hline $8 d^{2} D_{5 / 2}-11 f^{2} F_{5 / 2}^{0}$ & $1.93 \times 10^{-5}$ & $4.28 \times 10^{-3}$ \\
\hline $9 d^{2} D_{3 / 2}-4 f^{2} F_{5 / 2}^{0}$ & $5.73 \times 10^{-5}$ & $1.87 \times 10^{-4}$ \\
\hline $9 d^{2} D_{3 / 2}-5 f^{2} F_{5 / 2}^{0}$ & $8.75 \times 10^{-5}$ & $9.43 \times 10^{-4}$ \\
\hline $9 d^{2} D_{3 / 2}-6 f^{2} F_{5 / 2}^{0}$ & $1.03 \times 10^{-4}$ & $3.59 \times 10^{-3}$ \\
\hline $9 d^{2} D_{3 / 2}-7 f^{2} F_{5 / 2}^{0}$ & $1.14 \times 10^{-4}$ & $1.47 \times 10^{-2}$ \\
\hline $9 d^{2} D_{3 / 2}-8 f^{2} F_{5 / 2}^{0}$ & $1.27 \times 10^{-4}$ & $1.01 \times 10^{-1}$ \\
\hline $9 d^{2} D_{3 / 2}-9 f^{2} F_{5 / 2}^{0}$ & $4.35 \times 10^{-8}$ & $1.78 \times 10^{-1}$ \\
\hline $9 d^{2} D_{3 / 2}-10 f^{2} F_{5 / 2}^{0}$ & $2.41 \times 10^{-4}$ & $7.67 \times 10^{-1}$ \\
\hline $9 d^{2} D_{3 / 2}-11 f^{2} F_{5 / 2}^{0}$ & $1.92 \times 10^{-4}$ & $2.07 \times 10^{-1}$ \\
\hline $9 d^{2} D_{5 / 2}-4 f^{2} F_{5 / 2}^{0}$ & $2.73 \times 10^{-6}$ & $1.34 \times 10^{-5}$ \\
\hline $9 d^{2} D_{5 / 2}-4 f^{2} F_{7 / 2}^{0}$ & $5.46 \times 10^{-5}$ & $2.01 \times 10^{-4}$ \\
\hline $9 d^{2} D_{5 / 2}-5 f^{2} F_{5 / 2}^{0}$ & $4.17 \times 10^{-6}$ & $6.73 \times 10^{-5}$ \\
\hline $9 d^{2} D_{5 / 2}-5 f^{2} F_{7 / 2}^{0}$ & $8.33 \times 10^{-5}$ & $1.01 \times 10^{-3}$ \\
\hline $9 d^{2} D_{5 / 2}-6 f^{2} F_{5 / 2}^{0}$ & $4.92 \times 10^{-6}$ & $2.56 \times 10^{-4}$ \\
\hline $9 d^{2} D_{5 / 2}-6 f^{2} F_{7 / 2}^{0}$ & $9.83 \times 10^{-5}$ & $3.85 \times 10^{-3}$ \\
\hline $9 d^{2} D_{5 / 2}-7 f^{2} F_{5 / 2}^{0}$ & $5.45 \times 10^{-6}$ & $1.05 \times 10^{-3}$ \\
\hline $9 d^{2} D_{5 / 2}-7 f^{2} F_{7 / 2}^{0}$ & $1.09 \times 10^{-4}$ & $1.58 \times 10^{-2}$ \\
\hline $9 d^{2} D_{5 / 2}-8 f^{2} F_{5 / 2}^{0}$ & $6.04 \times 10^{-6}$ & $7.22 \times 10^{-3}$ \\
\hline $9 d^{2} D_{5 / 2}-8 f^{2} F_{7 / 2}^{0}$ & $1.21 \times 10^{-4}$ & $1.08 \times 10^{-1}$ \\
\hline $9 d^{2} D_{5 / 2}-9 f^{2} F_{5 / 2}^{0}$ & $3.11 \times 10^{-9}$ & $8.46 \times 10^{-3}$ \\
\hline $9 d^{2} D_{5 / 2}-9 f^{2} F_{7 / 2}^{0}$ & $4.66 \times 10^{-8}$ & $1.69 \times 10^{-1}$ \\
\hline $9 d^{2} D_{5 / 2}-10 f^{2} F_{5 / 2}^{0}$ & $1.72 \times 10^{-5}$ & $3.65 \times 10^{-2}$ \\
\hline $9 d^{2} D_{5 / 2}-10 f^{2} F_{7 / 2}^{0}$ & $2.58 \times 10^{-4}$ & $7.30 \times 10^{-1}$ \\
\hline $9 d^{2} D_{5 / 2}-11 f^{2} F_{5 / 2}^{0}$ & $1.37 \times 10^{-5}$ & $9.84 \times 10^{-3}$ \\
\hline $10 d^{2} D_{3 / 2}-4 f^{2} F_{5 / 2}^{0}$ & $3.95 \times 10^{-5}$ & $1.18 \times 10^{-4}$ \\
\hline $10 d^{2} D_{3 / 2}-5 f^{2} F_{5 / 2}^{0}$ & $5.91 \times 10^{-5}$ & $5.41 \times 10^{-4}$ \\
\hline $10 d^{2} D_{3 / 2}-6 f^{2} F_{5 / 2}^{0}$ & $6.79 \times 10^{-5}$ & $1.77 \times 10^{-3}$ \\
\hline $10 d^{2} D_{3 / 2}-7 f^{2} F_{5 / 2}^{0}$ & $7.25 \times 10^{-5}$ & $5.55 \times 10^{-3}$ \\
\hline $10 d^{2} D_{3 / 2}-8 f^{2} F_{5 / 2}^{0}$ & $7.68 \times 10^{-5}$ & $2.03 \times 10^{-2}$ \\
\hline $10 d^{2} D_{3 / 2}-9 f^{2} F_{5 / 2}^{0}$ & $8.26 \times 10^{-5}$ & $1.29 \times 10^{-1}$ \\
\hline
\end{tabular}

C - estimated accuracy $\leq 25 \%$ 
TABLE VIII

Transition probabilities and oscillator strengths for multiplets of BI and comparison with accepted values and other results.

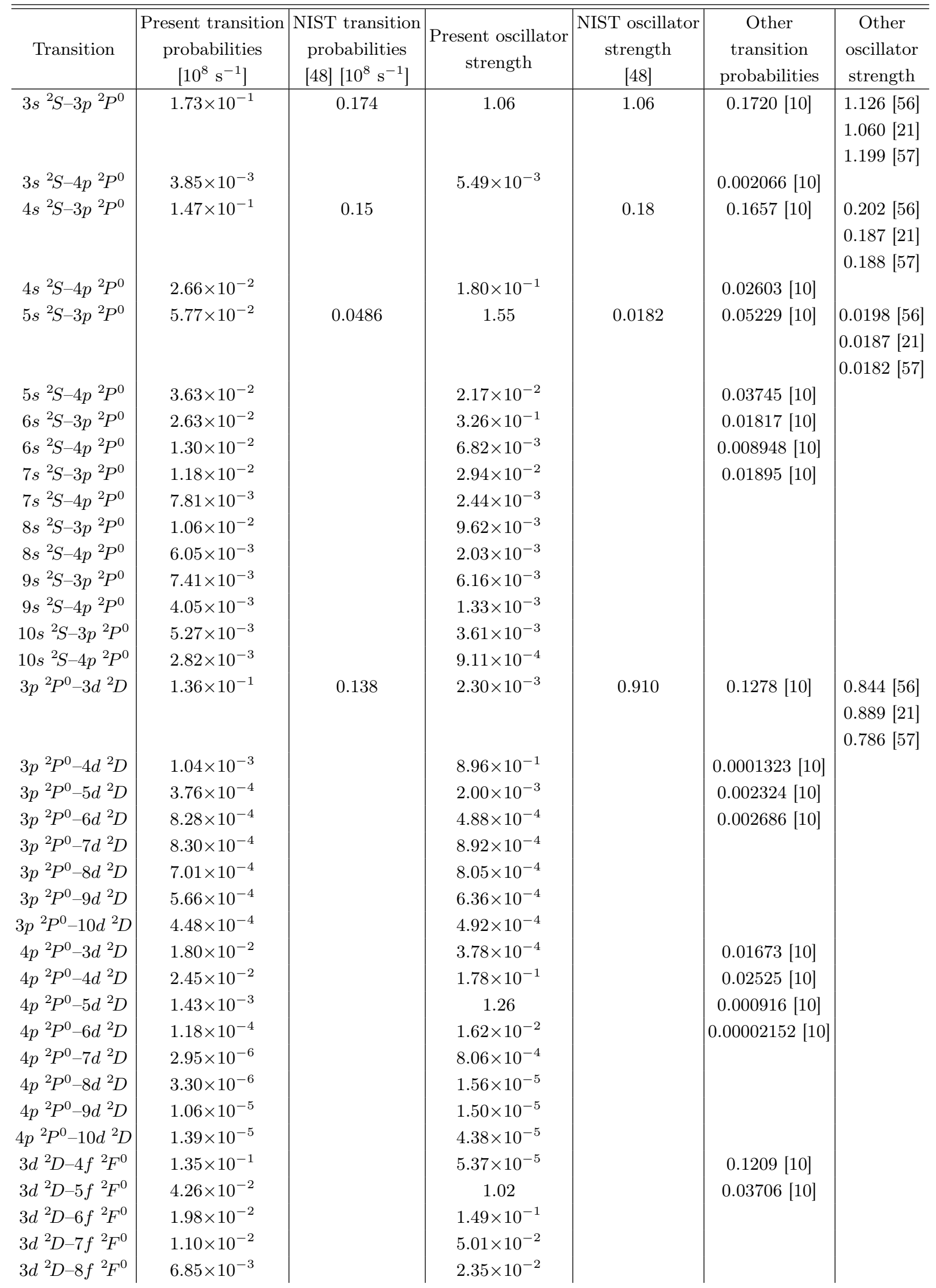


TABLE VIII (cont.)

\begin{tabular}{|c|c|c|c|c|c|c|}
\hline Transition & $\begin{array}{c}\text { Present transition } \\
\text { probabilities } \\
{\left[10^{8} \mathrm{~s}^{-1}\right]}\end{array}$ & $\begin{array}{c}\text { NIST transition } \\
\text { probabilities } \\
{[48]\left[10^{8} \mathrm{~s}^{-1}\right]}\end{array}$ & $\begin{array}{c}\text { Present oscillator } \\
\text { strength }\end{array}$ & \begin{tabular}{|c|} 
NIST oscillator \\
strength \\
{$[48]$} \\
\end{tabular} & $\begin{array}{c}\text { Other } \\
\text { transition } \\
\text { probabilities }\end{array}$ & $\begin{array}{c}\text { Other } \\
\text { oscillator } \\
\text { strength }\end{array}$ \\
\hline $3 d^{2} D-9 f^{2} F^{0}$ & $4.57 \times 10^{-3}$ & & $1.32 \times 10^{-2}$ & & & \\
\hline $3 d^{2} D-10 f^{2} F^{0}$ & $3.20 \times 10^{-3}$ & & $8.23 \times 10^{-3}$ & & & \\
\hline $3 d^{2} D-11 f^{2} F^{0}$ & $2.36 \times 10^{-3}$ & & $5.50 \times 10^{-3}$ & & & \\
\hline $4 d^{2} D-4 f^{2} F^{0}$ & $1.16 \times 10^{-7}$ & & $3.91 \times 10^{-3}$ & & $0.8309 \times 10^{-8}[10]$ & \\
\hline $4 d^{2} D-5 f^{2} F^{0}$ & $2.61 \times 10^{-2}$ & & $1.72 \times 10^{-2}$ & & $0.02470[10]$ & \\
\hline $4 d^{2} D-6 f^{2} F^{0}$ & $1.32 \times 10^{-2}$ & & $8.61 \times 10^{-1}$ & & & \\
\hline $4 d^{2} D-7 f^{2} F^{0}$ & $7.57 \times 10^{-3}$ & & $1.85 \times 10^{-1}$ & & & \\
\hline $4 d^{2} D-8 f^{2} F^{0}$ & $4.78 \times 10^{-3}$ & & $7.24 \times 10^{-2}$ & & & \\
\hline $4 d^{2} D-9 f^{2} F^{0}$ & $3.22 \times 10^{-3}$ & & $3.69 \times 10^{-2}$ & & & \\
\hline $4 d^{2} D-10 f^{2} F^{0}$ & $2.27 \times 10^{-3}$ & & $2.17 \times 10^{-2}$ & & & \\
\hline $4 d^{2} D-11 f^{2} F^{0}$ & $1.67 \times 10^{-3}$ & & $1.40 \times 10^{-2}$ & & & \\
\hline $5 d^{2} D-4 f^{2} F^{0}$ & $6.36 \times 10^{-4}$ & & $9.68 \times 10^{-3}$ & & $0.000627[10]$ & \\
\hline $5 d^{2} D-5 f^{2} F^{0}$ & $2.33 \times 10^{-7}$ & & $1.13 \times 10^{-2}$ & & $2.420 \times 10^{-8}[10]$ & \\
\hline $5 d^{2} D-6 f^{2} F^{0}$ & $7.15 \times 10^{-3}$ & & $5.08 \times 10^{-2}$ & & & \\
\hline $5 d^{2} D-7 f^{2} F^{0}$ & $4.40 \times 10^{-3}$ & & $7.87 \times 10^{-1}$ & & & \\
\hline $5 d^{2} D-8 f^{2} F^{0}$ & $2.83 \times 10^{-3}$ & & $1.92 \times 10^{-1}$ & & & \\
\hline $5 d^{2} D-9 f^{2} F^{0}$ & $1.93 \times 10^{-3}$ & & $8.02 \times 10^{-2}$ & & & \\
\hline $5 d^{2} D-10 f^{2} F^{0}$ & $1.37 \times 10^{-3}$ & & $4.25 \times 10^{-2}$ & & & \\
\hline $5 d^{2} D-11 f^{2} F^{0}$ & $1.01 \times 10^{-3}$ & & $2.57 \times 10^{-2}$ & & & \\
\hline $6 d^{2} D-4 f^{2} F^{0}$ & $2.76 \times 10^{-4}$ & & $1.70 \times 10^{-2}$ & & $0.0002630[10]$ & \\
\hline $6 d^{2} D-5 f^{2} F^{0}$ & $4.92 \times 10^{-4}$ & & $2.03 \times 10^{-3}$ & & $0.0005014[10]$ & \\
\hline $6 d^{2} D-6 f^{2} F^{0}$ & $1.77 \times 10^{-7}$ & & $2.99 \times 10^{-2}$ & & & \\
\hline $6 d^{2} D-7 f^{2} F^{0}$ & $2.51 \times 10^{-3}$ & & $8.39 \times 10^{-2}$ & & & \\
\hline $6 d^{2} D-8 f^{2} F^{0}$ & $1.73 \times 10^{-3}$ & & $7.57 \times 10^{-1}$ & & & \\
\hline $6 d^{2} D-9 f^{2} F^{0}$ & $1.19 \times 10^{-3}$ & & $1.95 \times 10^{-1}$ & & & \\
\hline $6 d^{2} D-10 f^{2} F^{0}$ & $8.55 \times 10^{-4}$ & & $8.43 \times 10^{-2}$ & & & \\
\hline $6 d^{2} D-11 f^{2} F^{0}$ & $6.33 \times 10^{-4}$ & & $4.57 \times 10^{-2}$ & & & \\
\hline $7 d^{2} D-4 f^{2} F^{0}$ & $1.47 \times 10^{-4}$ & & $2.81 \times 10^{-2}$ & & & \\
\hline $7 d^{2} D-5 f^{2} F^{0}$ & $2.42 \times 10^{-4}$ & & $7.31 \times 10^{-4}$ & & & \\
\hline $7 d^{2} D-6 f^{2} F^{0}$ & $3.17 \times 10^{-4}$ & & $5.61 \times 10^{-3}$ & & & \\
\hline $7 d^{2} D-7 f^{2} F^{0}$ & $1.15 \times 10^{-7}$ & & $5.31 \times 10^{-2}$ & & & \\
\hline $7 d^{2} D-8 f^{2} F^{0}$ & $1.05 \times 10^{-3}$ & & $1.16 \times 10^{-1}$ & & & \\
\hline $7 d^{2} D-9 f^{2} F^{0}$ & $7.71 \times 10^{-4}$ & & $7.47 \times 10^{-1}$ & & & \\
\hline $7 d^{2} D-10 f^{2} F^{0}$ & $5.62 \times 10^{-4}$ & & $1.98 \times 10^{-1}$ & & & \\
\hline $7 d^{2} D-11 f^{2} F^{0}$ & $4.18 \times 10^{-4}$ & & $8.73 \times 10^{-2}$ & & & \\
\hline $8 d^{2} D-4 f^{2} F^{0}$ & $8.79 \times 10^{-5}$ & & $4.79 \times 10^{-2}$ & & & \\
\hline $8 d^{2} D-5 f^{2} F^{0}$ & $1.38 \times 10^{-4}$ & & $3.53 \times 10^{-4}$ & & & \\
\hline $8 d^{2} D-6 f^{2} F^{0}$ & $1.70 \times 10^{-4}$ & & $2.06 \times 10^{-3}$ & & & \\
\hline $8 d^{2} D-7 f^{2} F^{0}$ & $1.99 \times 10^{-4}$ & & $1.03 \times 10^{-2}$ & & & \\
\hline $8 d^{2} D-8 f^{2} F^{0}$ & $7.30 \times 10^{-8}$ & & $7.94 \times 10^{-2}$ & & & \\
\hline $8 d^{2} D-9 f^{2} F^{0}$ & $4.95 \times 10^{-4}$ & & $1.47 \times 10^{-1}$ & & & \\
\hline $8 d^{2} D-10 f^{2} F^{0}$ & $3.83 \times 10^{-4}$ & & $7.51 \times 10^{-1}$ & & & \\
\hline $8 d^{2} D-11 f^{2} F^{0}$ & $2.89 \times 10^{-4}$ & & $2.03 \times 10^{-1}$ & & & \\
\hline $9 d^{2} D-4 f^{2} F^{0}$ & $5.73 \times 10^{-5}$ & & $8.99 \times 10^{-2}$ & & & \\
\hline $9 d^{2} D-5 f^{2} F^{0}$ & $8.75 \times 10^{-5}$ & & $2.01 \times 10^{-4}$ & & & \\
\hline $9 d^{2} D-6 f^{2} F^{0}$ & $1.03 \times 10^{-4}$ & & $1.01 \times 10^{-3}$ & & & \\
\hline $9 d^{2} D-7 f^{2} F^{0}$ & $1.14 \times 10^{-4}$ & & $3.85 \times 10^{-3}$ & & & \\
\hline
\end{tabular}


TABLE VIII (cont.)

\begin{tabular}{c|c|c|c|c|c|c}
\hline \hline Transition & $\begin{array}{c}\text { Present transition } \\
\text { probabilities } \\
{\left[10^{8} \mathrm{~s}^{-1}\right]}\end{array}$ & $\begin{array}{c}\text { NIST transition } \\
\text { probabilities } \\
{[48]\left[10^{8} \mathrm{~s}^{-1}\right]}\end{array}$ & $\begin{array}{c}\text { Present oscillator } \\
\text { strength }\end{array}$ & $\begin{array}{c}\text { NIST oscillator } \\
\text { strength } \\
{[48]}\end{array}$ & $\begin{array}{c}\text { Other } \\
\text { transition } \\
\text { probabilities }\end{array}$ & $\begin{array}{c}\text { Other } \\
\text { oscillator } \\
\text { strength }\end{array}$ \\
\hline $9 d^{2} D-8 f{ }^{2} F^{0}$ & $1.27 \times 10^{-4}$ & & $1.58 \times 10^{-2}$ & & \\
$9 d^{2} D-9 f{ }^{2} F^{0}$ & $4.66 \times 10^{-8}$ & & $1.08 \times 10^{-1}$ & & \\
$9 d^{2} D-10 f^{2} F^{0}$ & $2.58 \times 10^{-4}$ & & $7.78 \times 10^{-1}$ & & \\
$9 d^{2} D-11 f^{2} F^{0}$ & $2.06 \times 10^{-4}$ & & $2.07 \times 10^{-1}$ & & \\
$10 d^{2} D-4 f^{2} F^{0}$ & $3.95 \times 10^{-5}$ & & $1.26 \times 10^{-4}$ & & \\
$10 d^{2} D-5 f{ }^{2} F^{0}$ & $5.91 \times 10^{-5}$ & & $5.79 \times 10^{-4}$ & & \\
$10 d^{2} D-6 f{ }^{2} F^{0}$ & $6.79 \times 10^{-5}$ & & $1.90 \times 10^{-3}$ & & \\
$10 d^{2} D-7 f{ }^{2} F^{0}$ & $7.25 \times 10^{-5}$ & & $5.95 \times 10^{-3}$ & & \\
$10 d^{2} D-8 f{ }^{2} F^{0}$ & $7.68 \times 10^{-5}$ & & $2.18 \times 10^{-2}$ & & \\
$10 d^{2} D-9 f{ }^{2} F^{0}$ & $8.26 \times 10^{-5}$ & & $1.38 \times 10^{-1}$ & & \\
$10 d^{2} D-10 f{ }^{2} F^{0}$ & $2.59 \times 10^{-8}$ & & $1.96 \times 10^{-1}$ & & \\
$10 d^{2} D-11 f{ }^{2} F^{0}$ & $1.44 \times 10^{-4}$ & & & & \\
\end{tabular}

\section{References}

[1] G.M. Lawrence, B.D. Savage, Phys. Rev. 141, 67 (1966).

[2] T. Andersen, K.A. Jessen, G. Sørensen, Phys. Rev. 188, 76 (1969).

[3] D. Goorvitch, F.P.J. Valero, Astrophys. J. 171, 643 (1972).

[4] R.A. Roig, G. Tondello, J. Phys. B, Atom. Mol. Phys. 9/14, 2373 (1976).

[5] M. Rødbro, R. Bruch, P. Bisgaard, J. Phys. B, Atom. Mol. Phys. 12/15, 2413 (1979).

[6] H. Bergström, G.W. Faris, H. Hallstadius, H. Lundberg, A. Persson, C.-G. Wahlström, Z. Phys. D, At. Mol. Clusters 8, 17 (1988).

[7] K.K. Irikura, R.D. Johnson III, J.W. Hudgens, J. Opt. Soc. Am. B 10/5, 763 (1992).

[8] W.G. Lynam, P.K. Carroll, J.T. Costello, D. Evans, G. O'Sullivan, J. Phys. B, At. Mol. Opt. Phys. 25, 3963 (1992).

[9] W.L. Glab, A.M. Falleur, J. Quant. Spectrosc. Radiat. Transfer 73, 121 (2001).

[10] H. Lundberg, Z.S. Li, P. Jonsson, Phys. Rev. A 63, 032505 (2001).

[11] W. Dankwort, E. Trefftz, Astr. Astrophys. 65, 93 (1978).

[12] Mina Vajed-Samii, Dinh Ton-That, L. Armstrong, Jr, Phys. Rev. A 23, 3034 (1980).

[13] J. Carlsson, P. Jönsson, L. Sturesson, C. Froese Fischer, Phys. Rev. A 49, 3426 (1994).

[14] P. Jönsson, C.F. Fischer, M.R. Godefroid, J. Phys. B, At. Mol. Opt. Phys. 29, 2393 (1996).

[15] G. Tachiev, C. Froese Fischer, J. Phys. B, At. Mol. Opt. Phys. 33, 2419 (2000).

[16] C. Froese Fischer, G. Tachiev, At. Data. Nucl. Data Tables 87, 1 (2004).
[17] C. Laughlin, A. Dalgarno, Phys. Rev. A 8, 39 (1973).

[18] J.A. Fernley, A. Hibbert, A.E. Kingston, M.J. Seaton, J. Phys. B, At. Mol. Opt. Phys. 32, 5507 (1999).

[19] A.W. Weiss, Phys. Rev. 188/1, 199 (1969).

[20] R.K. Nesbet, Phys. Rev. A 14, 1065 (1976).

[21] M. Cohen, J. Nahon, J. Phys. B, At. Mol. Opt. Phys. 13, 4325 (1980).

[22] L. Liang, Y.C. Wang, J. Phys. B, At. Mol. Opt. Phys. 36, 4387 (2003).

[23] U.I. Safronova, J. Plasma Res. Series 7, 282 (2006).

[24] N.W. Zheng, T. Wang, Astrophys. J. Suppl. S 143, 231 (2002).

[25] N.W. Zheng, T. Zhou, R.Y. Yang, T. Wang, D.X. Ma, Chem. Phys. 258, 37 (2000).

[26] D.X. Ma, N.W. Zheng, J. Fan, J. Phys. Chem. Ref. Data 33, 1013 (2004).

[27] D.X. Ma, N.W. Zheng, J. Fan, Int. J. Quantum Chem. 105, 120 (2005).

[28] N.W. Zheng, T. Wang, R.Y. Yang, J. Chem. Phys. 113, 6169 (2000).

[29] N.W. Zheng, D.X. Ma, R.Y. Yang, J. Chem. Phys. 113, 1681 (2002).

[30] J. Fan, N.W. Zheng, Chem. Phys. Lett. 400, 273 (2004).

[31] Yang Zhihu, Su Youwu, Acta Photon. Sin. 25, 783 (1996).

[32] Yan Zhiyu, Chinese J. At. Mol. Phys. 11, 445 (1994).

[33] Chen Chang-Yuan, Shen Hong-Lan, Sun Guo-Yao, Acta Phys. Sin. 46, 1055 (1997).

[34] Chen Zi-dong, Chen Gang, Chinese J. Chem. Phys. 18, 983 (2005).

[35] G. Celik, E. Akin, H.S. Kilic, Eur. Phys. J. D 40, 325 (2006).

[36] G. Celik, J. Quant. Spectrosc. Radiat. Transfer 103, 578 (2007). 
[37] G. Celik, E. Akin, H.S. Kilic, Int. J. Quantum Chem. 107, 495 (2007).

[38] N.W. Zheng, Chin. Sci. Bull. 31, 1238 (1986); 32, 1263 (1987); 33, 40 (1988).

[39] N.W. Zheng, A New Outline of Atomic Theory, Jiang Su Education Press, Nan Jing 1988.

[40] N.W. Zheng, T. Wang, D.X. Ma, T. Zhou, J. Fan, Int. J. Quantum Chem. 98, 281 (2004).

[41] D.X. Ma, N.W. Zheng, J. Fan, Int. J. Quantum Chem. 105, 12 (2005).

[42] N.W. Zheng, Y.J. Sun, Sci. China (Series B) 43, 113 (2000).

[43] W.C. Martin, J. Opt. Soc. Am. 70, 784 (1980).

[44] R.M. Langer, Phys. Rev. 35, 649 (1930).

[45] F. Rohrlich, Astrophys. J. 129, 441 (1959); F. Rohrlich, Astrophys. J. 129, 449 (1959).

[46] G. Racah, Phys. Rev. 62, 438 (1942); G. Racah, Phys. Rev. 63, 367 (1943).

[47] W.G. Wang, L.Y. Wang, R.D. Wang, Chin. Sci. Bull. 16, 1231 (1990).
[48] J.R. Fuhr, W.C. Martin, A. Musgrove, J. Sugar, W.L. Wiese, NIST Atomic Spectroscopic Database, word wide web url: http://physics.nist.gov .

[49] B. Kundu, P.K. Mukherjee, Theor. Chim. Acta 66, 173 (1984).

[50] C.E. Theodosiou, Phys. Rev. A 30, 2881 (1984).

[51] M.B. Viswanath, K.D. Sen, Theor. Chim. Acta 76, 373 (1989).

[52] F.W. King, Phys. Rev. A 44, 3350 (1991).

[53] J.P. Desclaux, Comput. Phys. Commun. 1, 216 (1969).

[54] A. Lindgard, S.E. Nielsen, J. Phys. B, At. Mol. Phys. 8, 1183 (1975).

[55] A. Lindgard, S.E. Nielsen, At. Data Nucl. Data Tables 19, 533 (1977).

[56] R.P. McEachran, M. Cohen, J. Quant. Spectrosc. Radiat. Transfer 11, 1819 (1971).

[57] W.L. Wiese, M.W. Smith, B.M. Glennon, Atomic Transition Probabilities, Vol. I, US Govt Printing Office, Washington DC 1966. 